\title{
The CIP2A-TOPBP1 complex safeguards chromosomal stability during mitosis
}

Mara De Marco Zompit ${ }^{1}$, Clémence Mooser ${ }^{1}$, Salomé Adam², Silvia Emma Rossi², Alain Jeanrenaud $^{1}$, Pia-Amata Leimbacher ${ }^{1}$, Daniel Fink ${ }^{1}$, Daniel Durocher ${ }^{2,3}$ and Manuel Stucki ${ }^{1,{ }^{*}}$

${ }^{1}$ Department of Gynecology, University Hospital Zurich, University of Zurich, Wagistrasse 14, CH-8952 Schlieren, Switzerland

${ }^{2}$ Lunenfeld-Tanenbaum Research Institute, Mount Sinai Hospital, 600 University Avenue, Toronto, Ontario, Canada M5G 1X5s

${ }^{3}$ Department of Molecular Genetics, University of Toronto, 1 King's College Circle, Toronto, ON, M5S 1A8, Canada

*Correspondence should be addressed to M.S. (manuel.stucki@uzh.ch) 
The accurate repair of DNA double-strand breaks (DSBs), highly toxic DNA lesions, is crucial for genome integrity and is tightly regulated during the cell cycle. In mitosis, cells inactivate DSB repair in favor of a tethering mechanism that stabilizes broken chromosomes until they are repaired in the subsequent cell cycle phases. How this is achieved mechanistically is not yet understood, but the adaptor protein TOPBP1 is critically implicated in this process. Here, we identify CIP2A as a TOPBP1-interacting protein that regulates TOPBP1 localization specifically in mitosis. Cells lacking CIP2A display increased micronuclei formation, DSB repair defects and chromosomal instability. CIP2A is actively exported from the cell nucleus in interphase but, upon nuclear envelope breakdown at the onset of mitosis, gains access to chromatin where it forms a complex with MDC1 and TOPBP1 to promote TOPBP1 recruitment to sites of mitotic DSBs. Collectively, our data uncover CIP2A-TOPBP1 as a mitosis-specific genome maintenance complex. 
Failure to accurately repair DSBs leads to genome instability, cell death or cancer ${ }^{1}$. Cells repair DSBs by two distinct families of pathways: non-homologous end joining (NHEJ) pathways that are the only DSB repair pathways active in G1, and homologous recombination (HR) pathways that require a sister chromatid as template and thus only occur in S and G2 phases ${ }^{2}$. By activating the G2/M checkpoint, cells buy time to repair the majority of DNA breaks before entering mitosis. However, DSBs can also occur during cell division, for example as a result of under-replicated DNA regions at common fragile sites, which impede chromosome segregation and may ultimately cause DNA breakage in mitosis $^{3-5}$. Cells rewire their response to DSBs in mitosis, at least in part to prevent telomeric fusions ${ }^{6}$. There is evidence that, instead of repairing them, mitotic cells stabilize chromosomal breaks until they can be safely repaired in the subsequent cell cycle phases ${ }^{6-9}$. How this is achieved mechanistically is not yet understood, but what is clear is that the chromatin response to DSBs is truncated in mitosis ${ }^{8}$. Early events such as phosphorylation of $\mathrm{H} 2 \mathrm{AX}$ and recruitment of MDC1 are intact, but further downstream responses that in interphase regulate DSB repair pathway choice are severed by the inability of mitotic cells to recruit RNF8/RNF168, 53BP1 and BRCA1 ${ }^{6,8}$. Instead, MDC1 mediates the recruitment of TOPBP1 to mitotic DSB sites, a process that is critical for the maintenance of chromosomal stability ${ }^{10}$. TOPBP1 is a highly versatile adaptor protein implicated in several aspects of genome integrity maintenance ${ }^{11}$. It is composed of different domains and regions, including nine BRCT domains, and an ATR activation domain (AAD; see Fig. 1a). TOPBP1 undergoes prominent enrichment at DSB sites throughout the cell cycle by at least four distinct mechanisms that involve phosphorylation-dependent interactions of alternating combinations of its four phospho-binding BRCT domains (BRCT 1, 2, 5 and 7) with the adaptor proteins 53BP1, RAD9, Treacle and MDC1 12 10,13,14. TOPBP1 interacts with Casein kinase 2 (CK2) phosphorylated MDC1 via its N-terminal BRCT0-2 module. This interaction is not cell cycle 
regulated but paradoxically, MDC1 recruits TOPBP1 by direct interaction exclusively in mitosis ${ }^{10}$. Here we present a possible solution to this conundrum by identifying cancerous inhibitor of protein phosphatase 2A (CIP2A) as a TOPBP1-interacting protein that promotes TOPBP1 recruitment to sites of DSBs specifically in mitosis. We further demonstrate that loss of CIP2A leads to DSB repair defects, increased micronuclei formation and chromosomal instability. Finally, we propose that CIP2A-TOPBP1 complex formation is regulated during the cell cycle by nuclear export, which spatially sequesters CIP2A from TOPBP1 in interphase cells, thus allowing their efficient interaction exclusively in mitosis. 


\section{RESULTS}

\section{CIP2A is a TOPBP1 interaction partner.}

We previously demonstrated that TOPBP1 is recruited to sites of DSBs in mitosis by direct phosphorylation-dependent interactions between its N-terminal BRCT domains with phosphorylated MDC $1{ }^{10}$. Interestingly, we observed that a C-terminal TOPBP1 deletion mutant containing BRCT domains 0-5 but lacking BRCT domains 6-8 and the AAD was recruited to sites of DSBs in G1, but not in mitosis (Fig. 1b). This suggested that domains/regions downstream of BRCT5 are important for TOPBP1 DSB recruitment in mitosis. Inactivating point mutations in BRCT7 and the AAD, and deletion of BRCT6 did not affect TOPBP1 recruitment, neither in interphase nor in mitosis (Fig. 1b). However, deletion of a conserved region between BRCT5 and 6 (amino acids 813-892) abrogated mitotic DSB recruitment, while recruitment in interphase was unaffected (Fig. 1a,b and Supplementary Fig. 1a). To test if this region constitutes a previously uncharacterized interaction surface for a protein that mediates TOPBP1 recruitment specifically in mitosis, we expressed a hemagglutinin (HA)-tagged protein fragment corresponding to the entire region between BRCT5 and 6 (human TOPBP1 residues 740-899) in 293-T cells, followed by immunoprecipitation with HA affinity beads. Bound proteins were identified by liquid chromatography-tandem mass spectrometry (LC-MS/MS). Among the 16 proteins present only in the HA-TOPBP1(740-899) pull-down but not in the control, CIP2A (also called KIAA1524) caught our attention (Supplementary Fig. 1b,c). We previously identified this protein in a proteomic screen for interaction partners of full-length TOPBP $1{ }^{14}$. Moreover, it was also identified in a recent proximity proteomic screen for BRCA1-interacting factors along with TOPBP1 ${ }^{15}$. CIP2A was originally described as an endogenous inhibitory factor of protein phosphatase $2 \mathrm{a}(\mathrm{PP} 2 \mathrm{~A})$, and the protein is overexpressed in multiple cancers ${ }^{16,17}$. Interestingly, genotoxin sensitivity profiling by CRISPR-Cas9 drop-out screens revealed high 
sensitivity of CIP2A loss to ATR inhibitors and drugs that induce DSBs in proliferating cells such as Topoiomerase I and II inhibitors ${ }^{18,19}$. Inspection of one of these DNA damage chemogenetic datasets ${ }^{19}$ revealed a significant drug sensitivity correlation between MDC1 and CIP2A (Supplementary Fig. 1d). Combined, these were cues to further investigate a potential link between CIP2A and TOPBP1 in the mitotic DSB response. Western blotting confirmed that CIP2A was specifically pulled down by the conserved region between BRCT5 and 6 of TOPBP1 (Fig. 1c). In addition, co-immunoprecipitation with GFP-tagged full-length TOPBP1 and deletion mutants showed defective interaction with the C-terminal deletion mutant, and significantly reduced interaction upon deletion of amino acids 813-892 ( $\Delta 813$ 192; Fig. 1d). Together, these findings identify CIP2A as a TOPBP1 interacting factor that binds to a region in TOPBP1 that localizes between BRCT5 and 6.

\section{CIP2A interacts with TOPBP1 at sites of DSBs in mitosis.}

Consistent with a previous report ${ }^{20}$ we found that in undamaged cells, CIP2A is localizing to the cytoplasm in interphase and accumulates on centrosomes along with TOPBP1 in mitosis (Fig. 2a). Strikingly, upon irradiation, CIP2A was markedly enriched in foci on condensed mitotic chromosomes, where it co-localized with TOPBP1, while it was not detectable at sites of DSBs in interphase cells (Fig. 2b-d). By using high-resolution microscopy, we had previously observed that many IR-induced TOPBP1 structures in mitosis assumed the shape of filamentous assemblies ${ }^{10}$. Airyscan confocal microscopy showed that CIP2A was also present in such filaments on mitotic chromosomes and consistently co-localized with TOPBP1 (Fig. 2e). To test if CIP2A and TOPBP1 interact at sites of DSBs in mitosis we performed proximity ligation assays (in situ PLA). In unirradiated mitotic cells, the PLA signal was evenly distributed throughout the cell with occasional focal accumulation. Upon irradiation, the PLA signal was mostly concentrated in foci that colocalized with DAPI 
staining, indicating that CIP2A and TOPBP1 indeed interact at sites of DBSs on mitotic chromosomes (Fig. 2f). These data indicate that CIP2A is recruited to sites of DSBs exclusively in mitosis where it co-localizes with TOPBP1.

\section{CIP2A mediates TOPBP1 DSB accumulation in mitosis.}

Next, we sought to determine if CIP2A is required for TOPBP1 recruitment to DSBs on mitotic chromosomes. To this end, we knocked out endogenous CIP2A in the hTERT immortalized non-transformed human retinal pigmented epithelial cell line RPE-1, using CRIPSR/Cas9 (henceforth termed $\triangle$ CIP2A; Fig. 3a and Supplementary Fig. 2a,b). TOPBP1 foci were completely absent in irradiated mitotic $\triangle \mathrm{CIP} 2 \mathrm{~A}$ cells, but they were fully restored by stable transduction of $\triangle \mathrm{CIP} 2 \mathrm{~A}$ cells with a lentiviral vector containing Flag-tagged wild type CIP2A cDNA (Fig. 3b,c), thus ruling out off-target effects of the guide RNA. TOPBP1 enrichment at mitotic DSBs was also completely defective in U2OS cells in which CIP2A was depleted by siRNA transfection, indicating that this effect is not cell type specific (Supplementary Fig. 2c). Depletion of TOPBP1 by siRNA also abrogated CIP2A foci in mitotic cells, suggesting that for efficient accumulation at sites of mitotic DSBs, TOPBP1 and CIP2A may be dependent upon each other (Fig. 3d). However, we also noted that depletion of TOPBP1 led to a marked reduction of CIP2A expression, but not vice versa, which could also have contributed to the apparent reduction of CIP2A foci in TOPBP1 depleted mitotic cells (Supplementary Fig. 2d). To test if TOPBP1 must exist in a complex with CIP2A in order to be recruited to sites of mitotic chromosome breaks, we knocked-down TOPBP1 expression in U2OS cells by 3'-UTR-targeting siRNA followed by re-expression of GFP-tagged wild type or TOPBP1 $\Delta 813-192$. CIP2A only accumulated in foci in the presence of wild type TOPBP1, but not in the presence of the $\Delta 813-192$ mutant, thus indicating that the recruitment of both of these proteins to sites of mitotic DNA breaks is 
dependent on their efficient interaction (Fig. 3e). We conclude from these results that CIP2A controls TOPBP1 recruitment to sites of mitotic DSBs and that CIP2A and TOPBP1 recruitment in mitosis are interdependent.

\section{CIP2A-TOPBP1 recruitment to sites of mitotic DSBs is mediated by MDC1.}

We next investigated the role of MDC1 in the recruitment of CIP2A and TOPBP1 to sites of mitotic DSBs. We previously demonstrated that in mitosis, TOPBP1 recruitment is dependent on a direct interaction between its N-terminal BRCT1 and BRCT2 domains with the two conserved phosphorylated Serine residues S168 and S196, respectively ${ }^{10}$. CIP2A and TOPBP1 recruitment were significantly reduced, but not completely abrogated, in RPE-1 MDC1 knock-out cells (RPE-1 $\triangle \mathrm{MDC} 1$ ) and in RPE-1 H2AX ${ }^{\mathrm{S139A} / \mathrm{S} 139 \mathrm{~A}}$ knock-in cells, as well as in U2OS MDC1 knock-out cells (U2OS $\triangle \mathrm{MDC} 1$ ), indicating that the majority of IRinduced CIP2A/TOPBP1 structures on mitotic chromosomes are dependent on a $\gamma \mathrm{H} 2 \mathrm{AX}-$ MDC1 mediated recruitment mechanism (Fig. 4a,b and Supplementary Fig. 3a-c). Consistent with the notion that CIP2A acts downstream of $\gamma \mathrm{H} 2 \mathrm{AX}$ and $\mathrm{MDC} 1$ in the mitotic response to DSBs, MDC1 recruitment is not affected in RPE-1 $\triangle$ CIP2A cells (Supplementary Fig. 3d). To test if CIP2A exists in a complex with MDC1 and TOPBP1, we transfected 293FT cells with GFP-tagged MDC1, followed by immunoprecipitation with anti-GFP affinity beads. Both CIP2A and TOPBP1 co-immunoprecipitated with GFP-MDC1 (Fig. 4c). Similarly, both TOPBP1 and MDC1 co-immunoprecipitated with overexpressed Flag-tagged CIP2A, thus suggesting that CIP2A and TOPBP1 exist in a ternary complex with MDC1 in vitro (Fig. 4d). To confirm that CIP2A is in close proximity to MDC1 also in vivo we performed in situ PLA with antibodies against CIP2A and MDC1. Similar to the results of the PLA assays with antibodies against CIP2A and TOPBP1, a clear PLA signal was detectable in mitotic cells and this signal was enriched in foci upon irradiation (Fig. 4e). Importantly, we also observed 
that both CIP2A and TOPBP1 foci were absent from irradiated mitotic chromosomes in U2OS $\triangle$ MDC1 cells stably expressing GFP-tagged S168A/S196A MDC1, while they were readily detectable and co-localized with MDC1 in wild type GFP-MDC1 expressing cells (Fig. 4f,g). This indicates that accumulation of both CIP2A and TOPBP1 at sites of mitotic DSBs is dependent on the direct interaction between TOPBP1 BRCT1 and BRCT2 with phosphorylated MDC1. Notably, in CIP2A depleted cells, TOPBP1 interacted as efficiently with MDC1 as in CIP2A expressing control cells, indicating that CIP2A does not simply promote the association of TOPBP1 with MDC1 (Fig. 4h). In summary, our data suggest that TOPBP1 must exist in a complex with CIP2A in order to be efficiently recruited to sites of mitotic DSBs by $\gamma \mathrm{H} 2 \mathrm{AX}-\mathrm{MDC} 1$.

\section{CRM1-dependent nuclear export sequesters CIP2A from TOPBP1 in interphase cells.}

CIP2A is a 905 amino acid protein, roughly composed of two structurally distinct regions: a N-terminal Armadillo repeat domain (Arm; amino acids 1-560) and a C-terminal predicted coiled-coil region (Fig. 5a) ${ }^{21}$. In a proteomic screen, CIP2A was identified as a target of the chromosome region maintenance 1 (CRM1, also called exportin 1) transport receptor for the export of proteins from the nucleus ${ }^{22}$. Indeed, we observed that treatment of cells with two selective CRM1 inhibitors (Leptomycin B and Selinexor) led to a significant increase in nuclear localization of CIP2A in interphase cells (Fig. 5b,c). CRM1 binds to its targets via a flexible recognition motif called nuclear export signal (NES). Three NES were predicted in CIP2A based on the Eukaryotic Linear Motive Resource for Function Sites in Proteins $\left(\mathrm{ELM}^{23}\right)$. Two of them reside in the N-terminal Arm region that mostly localized to the nucleus, thus ruling them out as genuine NES (data not shown). The third one (amino acids 598-612) is localized between the Arm repeat domain and the predicted C-terminal coiled coil region (Fig. 5a), and deletion of a fragment comprising this sequence motif (amino acids 
561-625) significantly increased nuclear CIP2A concentration in interphase cells, indicating that CIP2A is a direct CRM1 ligand (Fig. 5d-f). Since TOPBP1 is predominantly nuclear, the active transportation of CIP2A from the nucleus to the cytoplasm suggested that these two proteins are spatially separated during interphase. Staining of CIP2A in cells expressing endogenously tagged Lamin A (LMNA, a component of the nuclear envelope) showed that CIP2A is not present on chromatin until nuclear export ceases upon nuclear envelope breakdown at the onset of mitosis (Fig. 5g). Consistent with the idea that CIP2A is sequestered from TOPBP1 in interphase cells, we only observed PLA signals with CIP2A and TOPBP1 antibodies in mitotic cells (Fig. 5h). Together, these data show that CIP2A and TOPBP1 are spatially separated in interphase cells by CRM1-mediated nuclear export of CIP2A.

\section{CIP2A-independent TOPBP1 foci formation in interphase cells.}

An important prediction from the spatial separation of CIP2A from TOPBP1 in interphase cells is that CIP2A is not implicated in TOPBP1 recruitment to sites of DSBs in interphase. Indeed, in response to DSB induction by IR, TOPBP1 foci formation in CIP2A deficient interphase RPE-1 and U2OS cells is indistinguishable from cells that express CIP2A (Fig. 6a,b and Supplementary Fig. 4a,b). Moreover, CIP2A is also not implicated in Treaclemediated TOPBP1 accumulation in nucleolar caps after targeted induction of rDNA breakage (Fig. 6c,d). It is currently unclear if CIP2A interferes with DSB responses in interphase cells and thus needs to be sequestered from chromatin by nuclear export. Its presence in the nucleus of interphase cells does not seem to negatively impact on TOPBP1 recruitment to sites of DSBs (Supplementary Fig. 4c). Interestingly, we observed recruitment of CIP2A to foci and co-localization with TOPBP1 in a subset of Leptomycin B or Selinexor treated interphase cells (Fig. 6e,f). This suggests that CIP2A, when forced in the nucleus in 
interphase cells, is capable to bind to TOPBP1 at sites of DSBs, arguing against the possibility that CIP2A-TOPBP1 association is directly regulated during the cell cycle, but rather suggests that the regulation occurs via spatial separation in interphase due to nuclear export.

\section{CIP2A deficient cells display increased micronuclei formation, chromosomal instability and DSB repair defects.}

Cells unable to recruit TOPBP1 in mitosis due to disrupted MDC1-TOPBP1 interaction show increased micronuclei $(\mathrm{MNi})$ formation and chromosomal instability ${ }^{10}$, most likely due to the inability to properly segregate acentric chromosome fragments ${ }^{24}$. In line with its capacity to control TOPBP1 localization in mitosis, CIP2A deficient cells also displayed increased spontaneous MNi formation, which was slightly increased after IR treatment, and was rescued by re-expression of wild type CIP2A (Fig. 7a). The majority of MNi stained negative for CENPA, a centromeric marker, indicating that they mostly contain acentric chromosome fragments (Fig. 7b). Consequently, we also observed a significantly increased number of abnormal structures in metaphase spreads of RPE-1 $\triangle$ CIP2A cells, but not in RPE-1 $\triangle \mathrm{CIP} 2 \mathrm{~A}$ cells stably transduced with wild type CIP2A (Fig. 7c,d). To explore whether loss of CIP2A affects DSB repair in the subsequent interphase after irradiation in mitosis, we first synchronized parental RPE-1 cells and $\triangle \mathrm{CIP} 2 \mathrm{~A}$ cells as well as $\triangle \mathrm{CIP} 2 \mathrm{~A}$ cells stably expressing Flag-tagged wild type CIP2A in mitosis followed by irradiation and release them from the mitotic block. $\gamma \mathrm{H} 2 \mathrm{AX}$ foci (a marker for unrepaired DSBs) were quantified $24 \mathrm{~h}$ post-irradiation. There was a significant increase in residual $\gamma \mathrm{H} 2 \mathrm{AX}$ foci in cells deficient for CIP2A 24 h post-irradiation and re-expression of wild type CIP2A rescued this phenotype (Fig. 7e,f). We conclude from these results that CIP2A controls TOPBP1 recruitment to sites of mitotic DSBs and that in its absence, cells accumulate MNi and chromosomal instability. 


\section{CONCLUSION}

In summary, our findings demonstrate that CIP2A functions downstream of MDC1 to mediate TOPBP1 recruitment to sites of chromosome breaks in mitosis, which is critical for proper segregation of acentric chromosome fragments. As CIP2A deficiency gives rise to DSB repair defects after irradiation of mitotic cells, it is possible that CIP2A mediates productive DSB repair by stabilizing and/or tethering broken chromosomes during cell division until they enter the subsequent cell cycle where these lesions can be repaired. We have also shown that the effect of CIP2A on TOPBP1 is restricted to mitosis by nuclear exclusion, which is similar to the spatial control of the GEN1 Holliday junction resolvase whose activity is needed at the onset of mitosis but causes elevated crossover formation when present in the nucleus in interphase ${ }^{25}$. Cancer cells frequently have to deal with remnants of replication problems from the preceding S-phase, either as a consequence of oncogene-driven cell cycles, or as a result of defective HR pathways due to the loss of tumor suppressor genes such as BRCA1 and BRCA2. We surmise that at least a subset of these replication intermediates will be converted to DSBs in mitosis and will thus require the CIP2A-TOPBP1 complex for their stabilization. Indeed, in a complementary work submitted elsewhere, we found that loss of CIP2A or disruption of the CIP2A-TOPBP1 complex is lethal in HRdeficient cells, most likely due to rampant mis-segregation of acentric chromatin fragments that occur as a result of aberrant DNA replication in these cells (Adam et al., submitted). Collectively, our data firmly establish CIP2A-TOPBP1 as a physiologically critical component of the cellular machinery that deals with DNA lesions specifically during mitosis. 


\section{METHODS}

\section{Cell culture}

293T, U2OS, RPE-1 hTERT and HeLa cells were cultured in Dulbecco's modified Eagle's medium (DMEM Gibco $\left.{ }^{\mathrm{TM}}, 61965026\right)$, supplemented with 10\% fetal calf serum (PAN Biotech, P40-37500), 2 mM L-glutamine and penicillin-streptomycin antibiotics under standard cell culture conditions in a $\mathrm{CO} 2$ incubator $\left(37^{\circ} \mathrm{C} ; 5 \% \mathrm{CO} 2\right)$. Stably transfected U2OS cell lines were cultured in the presence of $400 \mu \mathrm{g} / \mathrm{mL}$ Geneticin (G418 sulfate, Sigma, G-3632). RPE-1 $\triangle$ CIP2A cell line complemented with lentiviral plasmids were cultured in the presence of $50 \mu \mathrm{g} / \mathrm{mL}$ Nourseothricin dihydrogen sulfate (Roth, 3011). The generation of U2OS and RPE1 $\triangle \mathrm{MDC} 1$ knock-out cell lines and complemented derivatives as well as RPE1 H2AX ${ }^{\mathrm{S} 139 \mathrm{~A} / \mathrm{S} 139 \mathrm{~A}}$ knock-in cell lines were previously described ${ }^{10}{ }^{26}$. Irradiation of cells was done in a YXLON, Y.SMART583 custom-made X-ray device. All cell lines were regularly tested for mycoplasma contamination.

\section{Generation of a CIP2A knock-out RPE-1 cell line}

RPE1 hTERT cells were cultured overnight at a density of $0.1 \times 10^{6}$ with $1 \mathrm{~mL}$ DMEM growth medium (Gibco $\left.{ }^{\text {TM }}, 61965026\right)$, supplemented with $10 \%$ FBS (PAN Biotech, P4037500), and Antibiotic-Antimycotic (Gibco $\left.{ }^{\mathrm{TM}}, 15240062\right)$. For transfection, $375 \mathrm{ng}$ of synthetic sgRNA (TrueGuide ${ }^{\mathrm{TM}}$, CRISPR932782_SGM) along with $6 \mu$ L Cas9 mRNA (Invitrogen ${ }^{\mathrm{TM}}, \mathrm{A} 29378$ ), and $18 \mu \mathrm{L}$ of the MessengerMAX ${ }^{\mathrm{TM}}$ reagent (Invitrogen ${ }^{\mathrm{TM}}$, LMRNA015) were separately diluted in $300 \mu \mathrm{L}$ Opti-MEM ${ }^{\mathrm{TM}}$ I (Gibco $\left.{ }^{\mathrm{TM}}, 31985047\right)$ each. After 10 min of incubation at room temperature, both mixtures were combined and added to the plate at $100 \mu \mathrm{L}$ per well. The cells were kept in culture for another $48 \mathrm{~h}$, trypsinized and transferred to a 96-well plate in serial two-fold dilution, starting with 4'000 counts. Single 
clones were successively identified by eye under a transluminescence microscope and progressively expanded to larger culture volumes. One successful knock-out could be identified by means of immunofluorescence as well as western blotting total cell extracts. An Adenine insertion at position 5110 of the gene was confirmed by subcloning the targeted area into a pCR ${ }^{\mathrm{TM}} 4 \mathrm{Blunt}-\mathrm{TOPO}{ }^{\circledR}$ vector (Invitrogen $\left.{ }^{\mathrm{TM}}, 450031\right)$ and subsequent colony sequencing of transformed Stellar ${ }^{\mathrm{TM}}$ Competent Cells (Takara, 636766). gRNA sequence: GGTCTTCAAGTAGCTCTACA

\section{Generation of endogenous-tagged Clover-LMNA Knock-In cell lines}

The nuclear lamina protein LMNA was endogenously labeled, in both HeLa and U2OS cell lines, with the monomeric green fluorescent protein Clover as previously described ${ }^{27}$. Briefly, cells were co-transfected with a Clover donor plasmid, that incorporates sequence homology to the locus adjacent to LMNA-start on each side of the tag, and one that encodes for Cas9 along with a chimeric gRNA, targeting two sites within the same region The cultures were expanded to confluency, detached using trypsin-EDTA (Gibco, 15400054), washed, and resuspended in $1.5 \mathrm{~mL}$ PBS buffer (Gibco, 10010015), supplemented with $1 \%$ BSA (Sigma-Aldrich, A7906), 25 mM HEPES (Gibco, 15630056), and 1 mM EDTA (AppliChem, A4892). The suspension was then passed through the cell strainer cap of a $5 \mathrm{~mL}$ PS test tube (Falcon, 352235). Single cells expressing relevant amounts of green fluorescence were isolated and collected in a cell sorter (BD Biosciences, FACSAria III 4L).

\section{Cloning and Mutagenesis}

pIRESneo2-EGFP-TOPBP1 plasmids are described in ${ }^{12}$. pIRESneo2-EGFP-TOPBP1$\triangle \mathrm{BRCT6}$ was generated by site directed mutagenesis of pIRESneo2-EGFP-TOPBP1-WT using QuikChange II XL Site-Directed Mutagenesis kit (Agilent Technologies, 200522). 
Sequences of the mutagenesis primers for TOPBP1- $\triangle \mathrm{BRCT} 6$ were, forward: 5'-CCC AAA ATG AGC TTG GAT ATC AGC GCA-3'; reverse:

5'- TTC CTT CTC TGA CTG GGC CTC TTT CAG-3'. Generation of pIRESneo2-EFGPTOPBP1-W1145R is described elsewhere ${ }^{14}$. pIRESneo2-EGFP-TOPBP1- $\triangle 813-892$ was generated by cloning a fragment of TOPBP1 lacking aa 813 to aa 892 that was generated synthetically by Genscript (genscript.com) into pIRESneo2-EGFP-TOPBP1-WT by using SbfI and StuI restriction enzymes. pHIV-NAT-T2A plasmids containing Flag-tagged fulllength CIP2A cDNA are described in Adam et al. (submitted). Deletion of the residues 561625 ( $\triangle$ NES) was done by deletion PCR using Phusion ${ }^{\circledR}$ High-Fidelity DNA Polymerase (NEB), followed by DpnI digestion of the template and transformation into bacteria. Primers were designed using the Quick Change primer design tool (Agilent) and here are the sequences: forward: 5'-AAA CAA TGC CTA TAG ACA ACA GGA ATA TGA AAT GAA ACT ATC CAC ATT AG-3'; reverse:

5'- CTA ATG TGG ATA GTT TCA TTT CAT ATT CCT GTT GTC TAT AGG CAT TGT TT-3'.

Plasmids pIRES V5 I-Ppo1 for I-Ppo1 mRNA production and purification were described ${ }^{28}$

\section{Lentivirus production}

293 T cells were plated at $80 \%$ confluence in a $10 \mathrm{~cm}$ dish with $8 \mathrm{~mL}$ of culture medium one day prior to transfection. Transfection was carried out using Lenti-X Packaging Single Shot (VSV-G) system (Takara Bio, 631275) according to the following protocol: $7 \mu \mathrm{g}$ of lentiviral plasmid were diluted in $600 \mu \mathrm{L}$ of sterile water, incubated at room temperature for $10 \mathrm{~min}$ and added to the cells dropwise. 293T infected cells were supplemented with fresh culture medium $4 \mathrm{~h}$ after infection and further incubated for 48 and $72 \mathrm{~h}$ before proceeding with the first and second lentivirus harvest, respectively. Lentivirus-containing supernatants were centrifuged at $4{ }^{\circ} \mathrm{C}$ for $10 \mathrm{~min}$ at $500 \mathrm{~g}$ and passed through a $0.45 \mu \mathrm{m}$ filter. Virus titer was 
measured using the QuickTiter ${ }^{\mathrm{TM}}$ Lentivirus Titer Kit (Cell Biolabs, VPK-107-T) according to the manufacturer's instructions.

\section{Transduction of RPE-1 $\Delta$ CIP2A cells}

RPE-1 $\triangle \mathrm{CIP} 2 \mathrm{~A}$ cells were plated in $6 \mathrm{~cm}$ dishes at $80 \%$ confluence in culture medium one day prior to infection. Cells were incubated with virus-containing supernatants in the presence of $8 \mu \mathrm{g} / \mathrm{mL}$ Polybrene (Santa Cruz Biotech, sc-134220) for $24 \mathrm{~h}$. The virus volume needed for infection was calculated using the following formula: Total Transduction Units $(\mathrm{TU})$ needed $=$ cells seeded $*$ MOI (in this experiment MOI $=5$ was used).

Total $\mathrm{mL}$ of lentiviral particles to add to each $6 \mathrm{~cm}$ dish $=($ Total $\mathrm{TU}$ needed $) /(\mathrm{TU} / \mathrm{mL})$ After incubation, virus-containing medium was discarded and replaced with fresh culture medium. Cells were incubated for an additional $48 \mathrm{~h}$ before proceeding with protein analysis to evaluate expression of the gene of interest. Transduced cells were selected in the presence of $100 \mu \mathrm{g} / \mathrm{mL}$ Nourseothricin dihydrogen sulfate.

\section{Cell transfection with RNA}

The control siRNA (siCtrl), siRNA targeting TOPBP1 (siTOPBP1) and the 3' UTR sequence of TOPBP1 gene (siTOPBP1 3'UTR) were obtained from Microsynth AG. The sequences of the siRNAs are the following:

siCtrl: UGGUUUACAUGUCGACUAA-dTdT, siTOPBP1: ACAAAUACAUGGCUGGUUA-dTdT

siTOPBP1 3'UTR: GUAAAUAUCUGAAGCUGUATT-dTdT

ON-Target plus SMART pool siRNA targeting CIP2A was purchased from Dharmacon (L014135-01-0005) and contains a mix of four different siRNAs:

Sequence 1: ACAGAACUCACACGACUA 
Sequence 2: GUCUAGGAUUAUUGGCAAA

Sequence 3: GAACAAAGGUUGCAGAUUC

Sequence 4: GCAGAGUGAUAUUGAGCAU

For siRNA transfection, cells were grown in a six-well plate $24 \mathrm{~h}$ prior to transfection. $60 \%$ confluent cells were transfected using $20 \mathrm{nM}$ siRNA and Lipofectamine RNAiMAX (Invitrogen, 13778150) according to the manufacturer's instructions. Cells were incubated with siRNA-lipid complexes for $48 \mathrm{~h}$ before being seeded on coverslips and harvested $72 \mathrm{~h}$ after transfection. I-Ppo1 mRNA transfection was done using the Lipofectamine MessengerMax Reagent (Invitrogen ${ }^{\mathrm{TM}}$, LMRNA015) according to the manufacturer's protocol.

\section{SDS-PAGE and western blotting}

SDS-PAGE and western blotting were performed using 4-20\% Mini-PROTEAN TGX Stainfree Precast Gels (Bio-Rad, 4568094) and Trans-Blot Turbo $0.2 \mu \mathrm{m}$ nitrocellulose membranes (Bio-Rad. 1704158), respectively. Antibodies against the following antigens were used at the indicated dilutions: GFP (Mouse, 11814460001, Roche, 1/5000), HA (Rabbit, ab9110, Abcam, 1/4000), MDC1 (Rabbit, ab11171, Abcam, 1/5000), TOPBP1 (Rabbit, ab2402, Abcam, 1/1500) and CIP2A (Rabbit, 14805, Cell Signaling, 1/1000). Blots were developed with SuperSignal ${ }^{\mathrm{TM}}$ West Femto Maximum Sensitivity Substrate (Thermo Scientific $^{\mathrm{TM}}$, 34096) and image acquisition was carried out using ChemiDoc MP Imaging System (Bio-Rad, 17001402).

\section{HA Immunoprecipitation}

For preparation of lysates for immunoprecipitations (IPs), cells were washed in cold PBS buffer pH 7.45 (Gibco $\left.^{\mathrm{TM}}, 18912-014\right)$, harvested with a cell scraper and centrifuged at $500 \mathrm{~g}$ 
for 3 min. PBS was then discarded and cells were resuspended in an appropriate volume of IP lysis buffer (50mM Tris- $\mathrm{HCl} \mathrm{pH} 7.6,150 \mathrm{mM} \mathrm{NaCl}, 5 \mathrm{mM} \mathrm{MgCl} 2,0.5 \%$ Triton X-100), supplemented with cOmplete EDTA-free protease inhibitor cocktail (Roche, 11873580001) and $25 \mathrm{U} / \mathrm{ml}$ Benzonase nuclease (Sigma, E1014). Cells were incubated on ice for $30 \mathrm{~min}$ and lysates were cleared by centrifugation at $16,000 \mathrm{~g}$ for $15 \mathrm{~min} .2 \mathrm{mg}$ of the soluble protein fraction were then transferred into fresh tubes and incubated with $30 \mu \mathrm{L}$ of monoclonal antiHA agarose beads (Sigma, A2095). Samples were incubated for $2 \mathrm{~h}$ with end-over-end rotation at $4{ }^{\circ} \mathrm{C}$. Immunoglobulin-antigen complexes were washed $3 \times 15$ min in cold PBS with end-over-end rotation before elution in 2X SDS sample buffer (Geneaid, PLD001, 100 mM DTT).

\section{GFP Immunoprecipitation}

Preparation of lysates was performed as described for HA-immunoprecipitation. $1 \mathrm{mg}$ of lysate was incubated with $25 \mu \mathrm{L}$ of equilibrated GFP-trap magnetic agarose beads (Chromotek, gtma-20) for $1 \mathrm{~h}$ with end-over-end rotation at $4{ }^{\circ} \mathrm{C}$. Samples were washed $4 \mathrm{x}$ 10 min in cold PBS with end-over-end rotation before elution in 2X SDS sample buffer.

\section{FLAG Immunoprecipitation}

For preparation of lysates, cells were washed in cold PBS buffer $\mathrm{pH} 7.45$, scraped and centrifuged at $500 \mathrm{~g}$ for $3 \mathrm{~min}$. PBS was then discarded and cells were resuspended in an appropriate volume of IP lysis buffer (50 mM Tris-HCl pH 7.4, $150 \mathrm{mM} \mathrm{NaCl,} 1 \mathrm{mM}$ EDTA, $1 \%$ Tryton X-100) supplemented with cOmplete EDTA-free protease inhibitor cocktail and $25 \mathrm{U} / \mathrm{mL}$ Benzonase nuclease. Lysates were cleared by centrifugation and $400 \mu \mathrm{g}$ of protein lysate were incubated with $40 \mu \mathrm{L}$ of Anti-FLAG ${ }^{\circledR}$ M2 affinity gel (Sigma, A2220) for $2 \mathrm{~h}$ 
with end-over-end rotation at $4{ }^{\circ} \mathrm{C}$. Immunoglobulin antigen complexes were washed $3 \times 15$ min in cold TBS with end-over-end rotation before elution in 2X SDS sample buffer.

\section{On-beads digestion and Mass Spectrometry}

The Functional Genomics Center of the University of Zurich (FGCZ) was commissioned to perform the mass spectrometry analysis. HA immunoprecipitation was performed as described above. After the last wash, precipitated material was resuspended in PBS buffer $\mathrm{pH}$ 7.45 and subjected to on-beads trypsin digestion according to the following protocol. Beads were washed twice with digestion buffer (10 mM Tris, $2 \mathrm{mM} \mathrm{CaCl2}, \mathrm{pH} 8.2)$. After the last wash, the buffer was discarded and monoclonal anti-HA agarose beads were resuspended in $10 \mathrm{mM}$ Tris, $2 \mathrm{mM} \mathrm{CaCl}$, $\mathrm{pH} 8.2$ buffer supplemented with trypsin $(100 \mathrm{ng} / \mu \mathrm{L}$ in $10 \mathrm{mM}$ $\mathrm{HCl})$. The $\mathrm{pH}$ was adjusted to 8.2 by the addition of $1 \mathrm{M}$ Tris $\mathrm{pH} 8.2$. Digestion was performed at $60{ }^{\circ} \mathrm{C}$ for $30 \mathrm{~min}$. Afterwards, supernatants were collected, and peptides were extracted from beads using $150 \mu \mathrm{L}$ of $0.1 \%$ trifluoroacetic acid (TFA). Digested samples were dried and reconstituted in $20 \mu \mathrm{L}$ ddH $2 \mathrm{O}+0.1 \%$ formic acid before performing Liquid chromatography-mass spectrometry analysis (LCMS/MS). For the analysis $1 \mu \mathrm{L}$ were injected on a nanoACQUITY UPLC system coupled to a Q-Exactive mass spectrometer (Thermo). MS data were processed for identification using the Mascot search engine (Matrixscience) and the spectra was searched against the Swissprot protein database (human and mice, retrieved 18/09/2020).

\section{Immunofluorescence}

Cells were grown on glass coverslips and washed two times with cold PBS before fixation with cold methanol for 10 min on ice. Methanol was discarded and cells were washed two times with PBS at room temperature and incubated with blocking buffer (10\% FCS in PBS) 
for at least 1 hour. Primary antibody incubations were performed at $4{ }^{\circ} \mathrm{C}$ overnight.

Coverslips were then washed 3x 10 min with PBS and secondary antibody incubation was performed for $1 \mathrm{~h}$ at room temperature in the dark. After washing 3x 10 min with PBS, coverslips were mounted on glass microscopy slides (Thermo Scientific, 630-1985, dimensions L76 X W26 mm) with VECTASHIELD ${ }^{\circledR}$ mounting medium containing 0.5 $\mu \mathrm{g} / \mathrm{mL}$ 4',6-diamidino-2-phenylindole dihydrochloride (DAPI) (Vector Laboratories, H1200). The following antibodies were used at the indicated dilutions: CIP2A (Mouse, sc80659, Santa Cruz, 1/800), Cyclin A (Mouse, 611269, BD Biosciences, 1/100), $\gamma \mathrm{H} 2 \mathrm{AX}$ (Mouse, 05-636-I, Merck, 1/500), MDC1 (Rabbit, ab11171, Abcam, 1/300), TOPBP1 (Rabbit, ABE1463, Millipore, 1/300) CENPA (Mouse, ab1339, Abcam, 1/500).

\section{Widefield microscopy}

Widefield image acquisition was done on a Leica DMI6000B inverted fluorescence microscope, equipped with Leica K5 sCMOS fluorescence camera (16-bit, 2048 x 2048 pixel, 4.2 MP) and Las X software version 3.7.2.22383. A HC Plan Apochromat 40X/0.95 PH dry objective and HCX Plan Apochromat 63X/1.40, and Plan Apochromat 100X/1.40 PH oil immersion objectives were used for image acquisition. For triple-wavelength emission detection, we combined DAPI with EGFP or Alexa Fluor 488 and Alexa Fluor 568.

\section{Confocal microscopy}

Confocal images were acquired with a Leica SP8 confocal laser scanning microscope coupled to a Leica DMI6000B inverted stand, with a 63x, 1.4-NA Plan-Apochromat oilimmersion objective. For quadruple-wavelength emission detection we combined DAPI with EGFP, Alexa Fluor 568 and Alexa Fluor 647. For triple-wavelength emission detection we combined DAPI with EGFP or Alexa Fluor 488, and Alexa Fluor 568. The sequential 
scanning mode was applied, and the number of overexposed pixels was kept at a minimum. 7 to10 z-sections were recorded with optimal distances based on Nyquist criterion. For optimal representation in figures, maximum intensity projections were calculated using Fiji ${ }^{29}$. For quantitative assessment of protein colocalization, the SQUASSH plugin (part of the MosaicSuite) for ImageJ and Fiji was used ${ }^{10,30}$.

\section{Image processing}

Unprocessed grayscale tagged image files (TIFs) and maximum intensity projections of confocal z-stacks were exported from Fiji, followed by pseudo-coloring and adjustment of exposure or brightness/contrast in Adobe Photoshop CC2020. For maximum data transparency and preservation, unprocessed grayscale images or maximum intensity projections were imported as smart objects and adjustments were done using adjustment layers. Processed images were saved as multilayer PSD files.

\section{Quantification of TOPBP1 foci in interphase cells}

Unsynchronized cells were grown on coverslips under standard culture conditions. Shortly before irradiation with $3 \mathrm{~Gy}$, the culture medium was changed and replaced with fresh medium containing either $10 \mu \mathrm{M}$ Leptomycin B (Apollo Scientific, BIL2101) or $10 \mu \mathrm{M}$ Selinexor (Focus Biomolecules, 10-4011-0005). For control cells, the medium was replaced with fresh medium containing DMSO. Irradiated cells were then further incubated for $3 \mathrm{~h}$ to achieve inhibition of CRM-1 mediated nuclear export of CIP2A and recruitment of TOPBP1 to DNA damage foci. Cells were then washed twice in cold PBS before fixation with ice-cold methanol and staining with anti-TOPBP1 and anti-CIP2A antibodies. Image acquisition was done using the Leica SP8 confocal microscope. Quantification was done using CellProfiler 
$3.0^{31}$ (CellProfiler analysis pipeline available upon request). The data was processed with $\mathrm{R}$ (r-project.org) and graphs were generated with GraphPad Prism 9.0

\section{Quantification of $\gamma \mathrm{H} 2 \mathrm{AX}$ foci}

For quantification of $\gamma \mathrm{H} 2 \mathrm{AX}$ foci, cells were grown on coverslips and incubated with RO3306 (Sigma, SML0569) for $16 \mathrm{~h}$ to synchronize cell population in late G2. Cells were subsequently released from the cell cycle arrest by washing $3 \mathrm{x} 5$ min with warm PBS and by adding fresh culture medium. Cells were further incubated for $20 \mathrm{~min}$ to allow them to progress into mitosis before irradiation with $0.5 \mathrm{~Gy}$, when indicated, and then fixed $24 \mathrm{~h}$ after irradiation with ice-cold methanol. Cells were stained with mouse monoclonal anti- $\gamma \mathrm{H} 2 \mathrm{AX}$ antibody as described above. Image acquisition was done using widefield microscopy and quantification was done using CellProfiler $3.0^{31}$ (CellProfiler analysis pipeline available upon request). The data was processed with $\mathrm{R}$ (r-project.org) and graphs were generated with GraphPad Prism 9.0

\section{Quantification of micronuclei}

For quantification of micronuclei formation after irradiation, unsynchronized cell populations were grown on coverslips and either treated with 3 Gy or left untreated. $6 \mathrm{~h}$ and $24 \mathrm{~h}$ after IR cells were washed in cold PBS and fixed with ice-cold methanol for $10 \mathrm{~min}$. Methanol was discarded and cells were washed twice before mounting the coverslips on microscopy glass slides with VECTRASHIELD containing DAPI. Images were captured by widefield microscopy and more then 1000 cells were taken into consideration for quantification. Micronuclei were counted manually in Fiji, and nuclei were counted using the CellProfiler 
3.0 software (CellProfiler analysis pipeline available upon request). For quantification of CENP-A positive and negative MNi, cells were stained with mouse monoclonal anti-CENPA antibody, following standard immunofluorescence protocol, as described above. Image acquisition was done using widefield microscopy and quantification of CENP-A positive and negative MNi was done manually.

\section{In Situ PLA assay}

U2OS cells were seeded on round glass coverslips (Thermo Scientific Menzel, CB00120RA120) in 24-well plates (TPP, 92024) at a density of $0.8 \times 10^{5}$ per well. When indicated, cells were incubated with 16.6 $\mu \mathrm{M}$ nocodazole (Sigma-Aldrich, M1404) for another $15 \mathrm{~h}$ to arrest them in prometaphase. Half of the samples were then irradiated with a dose of 1 Gy and placed back to the incubator for $1 \mathrm{~h}$. The growth medium was removed and all coverslips were washed with PBS, fixed with ice-cold methanol on ice for $12 \mathrm{~min}$, and washed again 3x in PBS. All subsequent steps were performed using Duolink® PLA Reagents (Sigma-Aldrich, DUO92001, DUO92005, DUO92008, DUO82049). The blocking solution was applied for $1 \mathrm{~h}$ in the humidified incubator at $37^{\circ} \mathrm{C}$. The following antibody combinations were used: mouse-anti-CIP2A (Santa Cruz, 2G10-35B; 1/800) and rabbit-antiTOPBP1 (Millipore, ABE1463; 1/250) or rabbit-anti-MDC1 (Abcam, ab11171; 1/200). Antibody incubation was done overnight at $4{ }^{\circ} \mathrm{C}$ under humid conditions. PLUS and MINUS PLA probes were prepared according to the manufacturer's protocol and applied analogous to the previous step but for $1 \mathrm{~h}$ at $37^{\circ} \mathrm{C}$ in the humidified incubator. Cells were washed for 15 min, then 10 min with wash buffer B, followed by 5 min with $0.01 \mathrm{X}$ wash buffer $\mathrm{B}$, and mounted on frosted glass slides using VECTASHIELD PLUS antifade mounting medium with DAPI (Adipogen, VC-H-2000). 


\section{Airyscan high-resolution confocal microscopy}

Cells were grown on high precision glass coverslips \#1.5H, $0.17 \mathrm{~mm}$ thick (Assistent) and either synchronized in late G2 with RO-3306, as described above or in prometaphase using $100 \mathrm{ng} / \mathrm{mL}$ nocodazole for $16 \mathrm{~h}$. Cells synchronized with RO-3306 were released into mitosis and irradiated with $1 \mathrm{~Gy}$. Irradiated cells were further incubated at $37^{\circ} \mathrm{C}$ and fixed at 30,45 and 60 min after irradiation in order to collect prophase, metaphase and anaphase cells, respectively. Nocodazole arrested cells were irradiated with $1 \mathrm{~Gy}$ and incubated for $1 \mathrm{~h}$ at $37^{\circ} \mathrm{C}$ before fixation. Cells were washed twice in cold PBS before fixation with ice-cold methanol for 10 min on ice. Methanol was discarded and cells were then washed twice with PBS at room temperature before incubation with blocking buffer (10\% FCS in PBS) for $2 \mathrm{~h}$. Primary antibodies were diluted in 5\% FCS in PBS and incubated overnight at $4{ }^{\circ} \mathrm{C}$. Cells were then washed 3x 10 min with blocking buffer before incubation with Alexa Fluor secondary antibodies (diluted 1/1000 in 5\% FCS) for $1 \mathrm{~h}$ at room temperature in the dark. Secondary antibody was then washed $3 \times 10$ min with PBS. Cells were then stained with 1 $\mu \mathrm{g} / \mathrm{mL}$ DAPI (AppliChem, A1001) diluted in PBS for $15 \mathrm{~min}$ at room temperature in the dark. DAPI was discarded and cells were further washed 2x 5 min with deionized water to completely remove the PBS. Coverslips were then mounted on microscopy glass slides without frosted edges (R. Langenbrick GmbH, Dimensions: L 76 x W 26 mm) using VECTASHIELD HardSet Antifade Mounting Medium (Vector Laboratories, H-1400). Airyscan confocal imaging was carried out at the ScopeM imaging facility of ETH Zurich, using an LSM 880 Airyscan inverted microscope (ZEISS) equipped with a DIC Plan Apochromat 63x/1.4 oil immersion objective and an Airyscan 32-pinhole detector unit. DAPI was detected using a 405-nm diode laser and 420-480 nm plus 495-550 nm band pass emission filters, Alexa 488 and GFP were detected with the 488-nm line of an argon laser and 
420-480 nm plus 495-550 nm band pass emission filters, and Alexa 568 was detected using a 561-nm diode-pumped solid-state laser and 495-550 nm band pass plus $570 \mathrm{~nm}$ long pass emission filters. Z-stacks of entire cells were acquired using optimal step size. Raw data were processed using Airyscan processing with Wiener Filter (auto setting) available in ZEN Black software version 2.1, yielding 8-bit images with approximately $180 \mathrm{~nm}$ lateral resolution. Display of images was adjusted for intensity for optimal display of structures of interest.

\section{Metaphase analysis}

Cells were grown to $90 \%$ confluence on a $10 \mathrm{~cm}$ plate and were treated with $0.1 \mu \mathrm{g} / \mathrm{mL}$ KaryoMax Colcemid (Thermo Fisher Scientific) for 16 h overnight. Cells were trypsinized and transferred to a $15 \mathrm{ml} \mathrm{Falcon}^{\mathrm{TM}}$ tube, centrifuged at $176 \mathrm{~g}$ for $5 \mathrm{~min}$ and carefully resuspended in $5 \mathrm{ml}$ of pre-warmed hypotonic buffer (15\% FBS, $75 \mathrm{mM} \mathrm{KCl})$ with intermittent agitation and incubated for $15 \mathrm{~min}$ at $37^{\circ} \mathrm{C}$. Cells were again pelleted at $176 \mathrm{~g}$ for $5 \mathrm{~min}$, the supernatant was discarded, and the cell pellet was resuspended in $200 \mu \mathrm{l}$ of hypotonic buffer. Cells were fixed by adding drop-wise $7 \mathrm{ml}$ of ice-cold $\mathrm{MeOH}: \mathrm{AcOH}$ 3:1 while slowly vortexing followed by $40 \mathrm{~min}$ on ice. After centrifugation at $176 \mathrm{~g}$ for $5 \mathrm{~min}$, supernatant was discarded, and cells were resuspended in remaining $200 \mu$ of the fixation buffer. $20-25 \mu 1$ of the cell suspension was then dropped at a $45^{\circ}$ angle onto a wet glass slide and air-dried for 10 min. Metaphases were stained with DAPI (VECTASHIELD containing DAPI), covered with a glass coverslip and sealed with nail varnish. Telomere FISH was conducted using the Telomere PNA FISH Kit/Cy3 (Dako, K5326) according to the protocol provided by the supplier. 


\section{REFERENCES}

1. Jackson, S. P. \& Bartek, J. The DNA-damage response in human biology and disease. Nature 461, 1071-1078 (2009).

2. Hustedt, N. \& Durocher, D. The control of DNA repair by the cell cycle. Nature cell biology 19, 1-9 (2017).

3. Fragkos, M. \& Naim, V. Rescue from replication stress during mitosis. Cell Cycle 16, 613-633 (2017).

4. Liu, Y., Nielsen, C. F., Yao, Q. \& Hickson, I. D. The origins and processing of ultra fine anaphase DNA bridges. Curr Opin Genet Dev 26, 1-5 (2014).

5. Mankouri, H. W., Huttner, D. \& Hickson, I. D. How unfinished business from S-phase affects mitosis and beyond. EMBO J 32, 2661-2671 (2013).

6. Orthwein, A. et al. Mitosis inhibits DNA double-strand break repair to guard against telomere fusions. Science (New York, NY) 344, 189-193 (2014).

7. Lukas, C. et al. 53BP1 nuclear bodies form around DNA lesions generated by mitotic transmission of chromosomes under replication stress. Nature Publishing Group 13, 243-253 (2011).

8. Giunta, S., Belotserkovskaya, R. \& Jackson, S. P. DNA damage signaling in response to double-strand breaks during mitosis. 190, 197-207 (2010).

9. Royou, A., Gagou, M. E., Karess, R. \& Sullivan, W. BubR1- and Polo-Coated DNA Tethers Facilitate Poleward Segregation of Acentric Chromatids. Cell 140, 235-245 (2010).

10. Leimbacher, P.-A. et al. MDC1 Interacts with TOPBP1 to Maintain Chromosomal Stability during Mitosis. Molecular Cell 74, 571-583.e8 (2019).

11. Wardlaw, C. P., Carr, A. M. \& Oliver, A. W. TopBP1: A BRCT-scaffold protein functioning in multiple cellular pathways. DNA Repair 22, 165-174 (2014).

12. Cescutti, R., Negrini, S., Kohzaki, M. \& Halazonetis, T. D. TopBP1 functions with 53BP1 in the G1 DNA damage checkpoint. The EMBO Journal 29, 3723-3732 (2010).

13. Bigot, N. et al. Phosphorylation-mediated interactions with TOPBP1 couple 53BP1 and 9-1-1 to control the G1 DNA damage checkpoint. eLife 8, 3894 (2019).

14. Mooser, C. et al. Treacle controls the nucleolar response to rDNA breaks via TOPBP1 recruitment and ATR activation. Nature Communications 11, 1-16 (2019).

15. Gupta, R. et al. DNA Repair Network Analysis Reveals Shieldin as a Key Regulator of NHEJ and PARP Inhibitor Sensitivity. Cell 173, 972-988.e23 (2018).

16. Junttila, M. R. et al. CIP2A inhibits PP2A in human malignancies. Cell 130, 51-62 (2007).

17. Khanna, A., Pimanda, J. E. \& Westermarck, J. Cancerous Inhibitor of Protein Phosphatase 2A, an Emerging Human Oncoprotein and a Potential Cancer Therapy Target. Cancer Research 73, 6548-6553 (2013).

18. Hustedt, N. et al. A consensus set of genetic vulnerabilities to ATR inhibition. Open Biology 9, 190156-190111 (2019).

19. Olivieri, M. et al. A Genetic Map of the Response to DNA Damage in Human Cells. Cell 182, 481-496.e21 (2020).

20. Kim, J. S., Kim, E. J., Oh, J. S., Park, I. C. \& Hwang, S. G. CIP2A modulates cell-cycle progression in human cancer cells by regulating the stability and activity of Plk1. Cancer Res 73, 6667-6678 (2013).

21. Wang, J. et al. Oncoprotein CIP2A is stabilized via interaction with tumor suppressor PP2A/B56. EMBO Rep 18, 437-450 (2017). 
22. Thakar, K., Karaca, S., Port, S. A., Urlaub, H. \& Kehlenbach, R. H. Identification of CRM1-dependent Nuclear Export Cargos Using Quantitative Mass Spectrometry. Molecular \& Cellular Proteomics 12, 664-678 (2013).

23. Dinkel, H. et al. ELM--the database of eukaryotic linear motifs. Nucleic Acids Res $\mathbf{4 0}$, D242-51 (2012).

24. Bizard, A. H. \& Hickson, I. D. ScienceDirect Anaphase: a fortune-teller of genomic instability. Current opinion in cell biology 52, 112-119 (2018).

25. Chan, Y. W. \& West, S. C. Spatial control of the GEN1 Holliday junction resolvase ensures genome stability. Nat Commun 5, 4844 (2014).

26. Chiang, T.-W. W., le Sage, C., Larrieu, D., Demir, M. \& Jackson, S. P. CRISPRCas9D10A nickase-based genotypic and phenotypic screening to enhance genome editing. Scientific reports 1-17 (2016).

27. Pinder, J., Salsman, J. \& Dellaire, G. Nuclear domain 'knock-in' screen for the evaluation and identification of small molecule enhancers of CRISPR-based genome editing. Nucleic acids research gkv993 (2015).

28. van Sluis, M. \& McStay, B. A localized nucleolar DNA damage response facilitates recruitment of the homology-directed repair machinery independent of cell cycle stage. Genes \& Development (2015).

29. Schindelin, J. et al. Fiji: an open-source platform for biological-image analysis. Nature Methods 9, 676-682 (2012).

30. Behan, F. M. et al. Prioritization of cancer therapeutic targets using CRISPR-Cas9 screens. Nature 568, 1-27 (2019).

31. McQuin, C. et al. CellProfiler 3.0: Next-generation image processing for biology. PLoS Biology 16, e2005970-17 (2018). 


\section{Acknowledgments}

We thank Thanos Halazonetis, Steve Jackson, Brian McStay and Graham Dellaire for providing valuable reagents, and Andrew Blackford for sharing unpublished observations. Imaging was performed with equipment maintained by the Center for Microscopy and Image Analysis, University of Zürich or the Scientific Center for Optical and Electron Microscopy (ScopeM) of the ETH Zürich. Cell sorting was carried out by the Flow Cytometry Core Facilities at the University of Zürich. Mass spectrometry was carried out by the Functional Genomics Center of the University of Zürich. The Durocher lab is supported by grants from the Canadian Institutes for Health Research (FDN143343) and the Canadian Cancer Society (705644). The Stucki lab is supported by two project grants from the Swiss National Foundation (31003A_163141 and 310030_189141) and by the Kanton of Zürich.

\section{Author contributions}

The project was conceived and supervised by M.S. and D.D. Biochemical and cell biological experiments were carried out and data were analyzed by M.D.Z., C.M., S.A., S.E.R., A.J., PA.L., D.F. and M.S. D.D. supervised S.A. and S.E.R. The paper was written by M.S. with contributions from other authors.

\section{Conflict of interest statement}

D.D. is a shareholder and advisor of Repare Therapeutics 
a

b
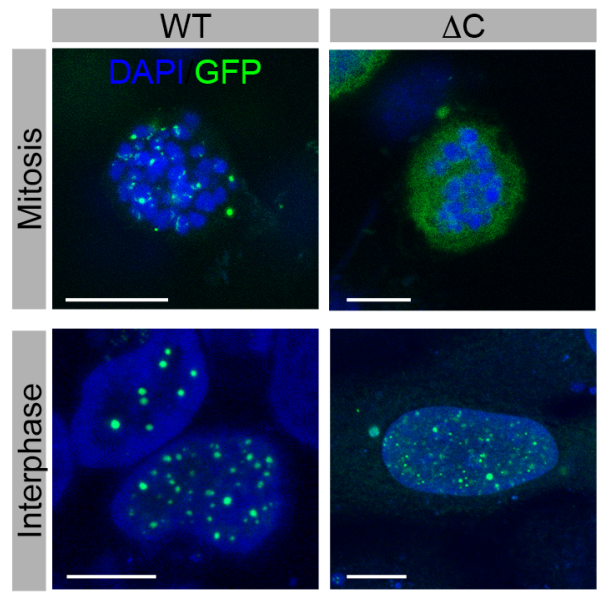

C

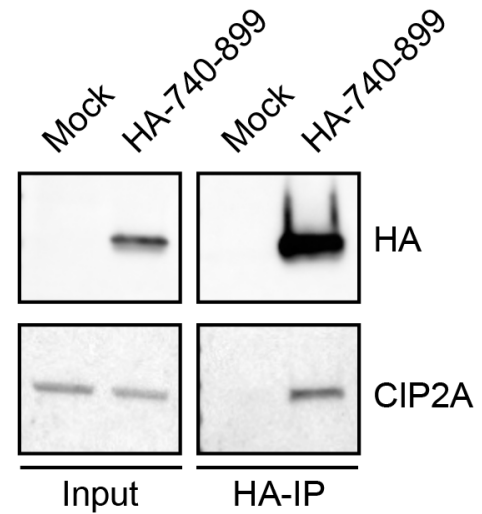

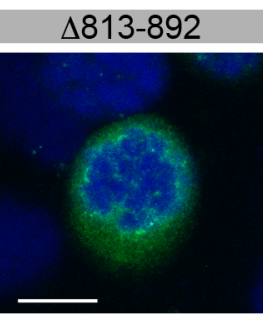
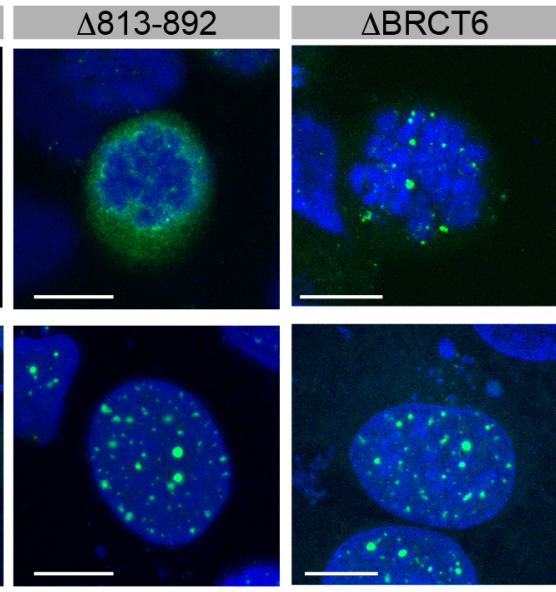

W1145 K1317

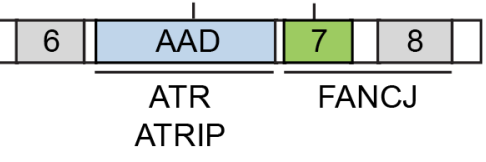

BLM

ATRIP

Treslin

Treacle

$\Delta \mathbf{C}$\begin{tabular}{|l|l|l|l|l|l|l||l|l|}
\hline 0 & 1 & 2 & & 3 & & 4 & 5 \\
\hline
\end{tabular}

\begin{tabular}{|c|c|c|c|c|c|c|c|c|c|c|}
\hline$\Delta 813-892$ & 0 & 1 & 2 & 3 & 4 & 5 & 6 & AAD & 7 & 8 \\
\hline$\triangle \mathrm{BRCT6}$ & 0 & 1 & 2 & 3 & 4 & 5 & & AAD & 7 & 8 \\
\hline
\end{tabular}
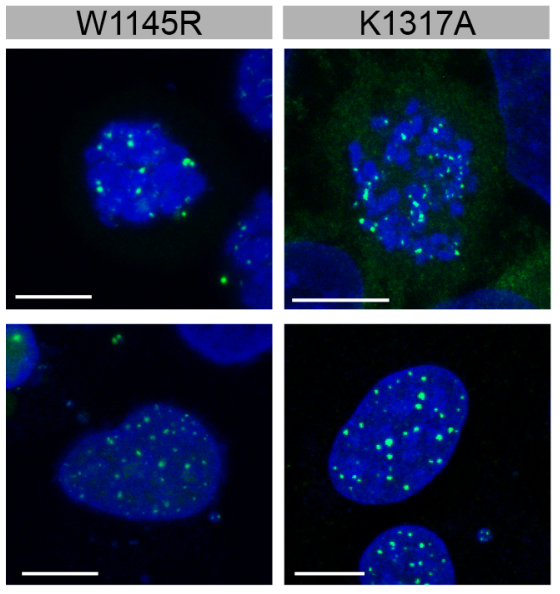

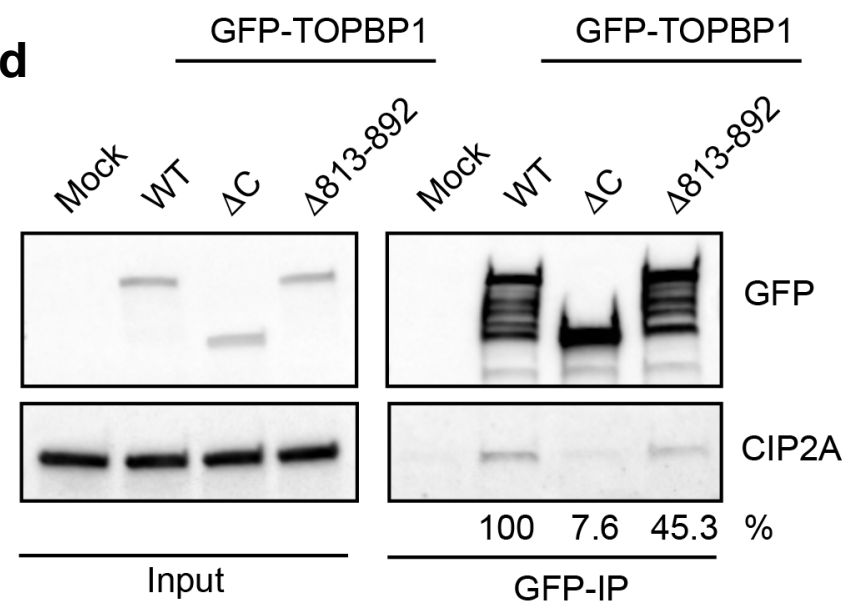

Fig. 1 CIP2A is a TOPBP1 interaction partner. a Schematic showing the layout of conserved domains and regions in TOPBP1. Key amino acids in the AAD (W1145) and BRCT7 (K1317) are indicated. GFP-tagged full-length TOPBP deletion constructs of TOPBP1, lacking either the C-terminal portion of the protein $(\triangle \mathrm{C})$, a conserved region between BRCT5 and 6 $(\triangle 813-892)$ and BRCT domain 6 ( $\triangle$ BRCT6). b Localization of GFP-TOPBP1 wild type and mutants in mitotic cells after $1 \mathrm{~Gy}$ of IR and interphase cells after $3 \mathrm{~Gy}$ of IR. Displayed are maximum intensity projections of confocal z-stacks. All scalebars $=10 \mu \mathrm{m}$. $\mathrm{c} \mathrm{HA}$-immunoprecipitation from 293FT cells transfected with a HA-tagged TOPBP1 fragment spanning the entire region between BRCT5 and 6 (amino acids 740-899). d GFP-immunoprecipitation from 293FT cells transfected with GFPtagged TOPBP1 wild type and indicated deletion mutants. Relative CIP2A band intensities are indicated. 
bioRxiv preprint doi: https://doi.org/10.1101/2021.02.08.430274; this version posted February 8, 2021. The copyright holder for this preprint (which was not certified by peer review) is the author/funder, who has granted bioRxiv a license to display the preprint in

a merge DAPI/CIP2A/TOPBP1

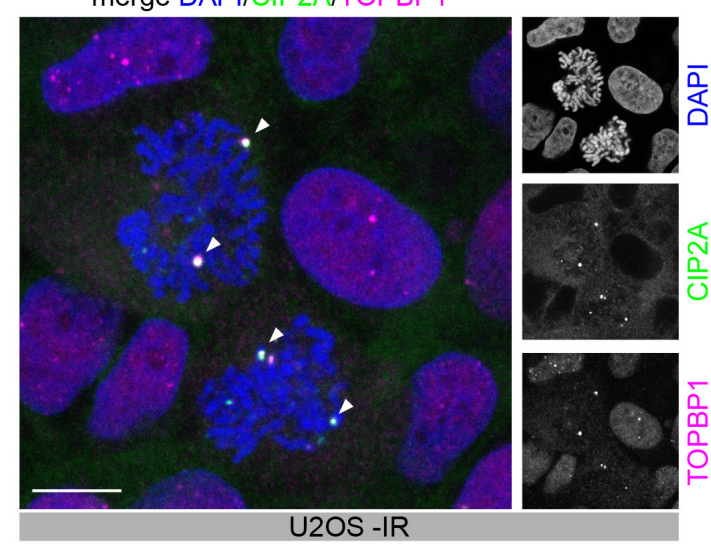

C

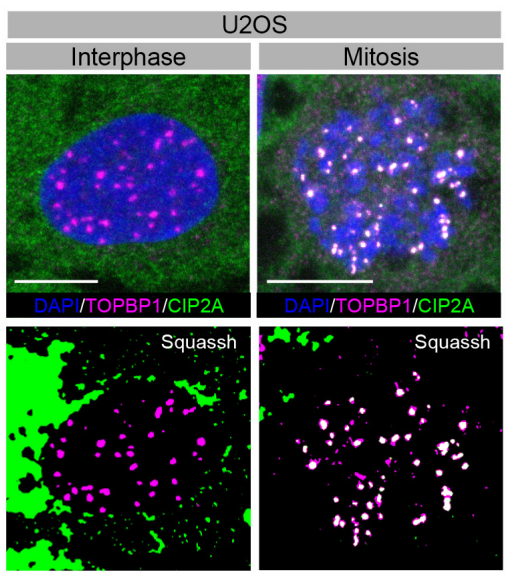

e
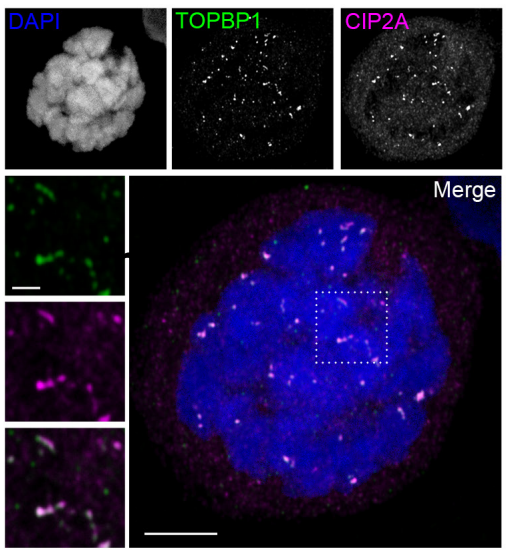

b

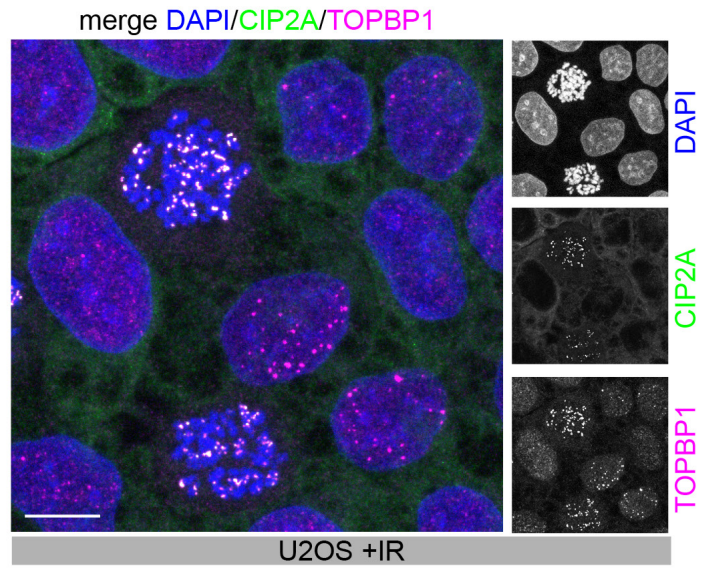

d
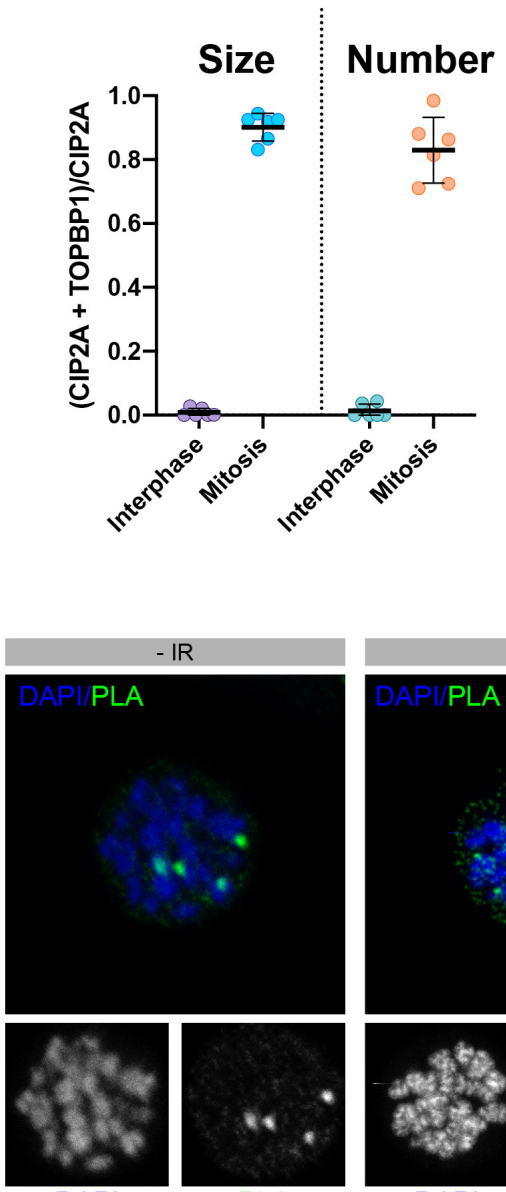

PLA

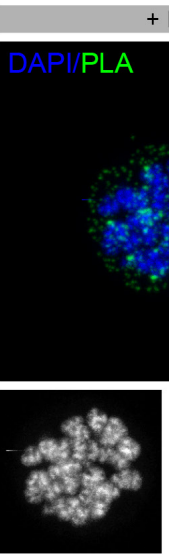

DAPI
$+\mathrm{IR}$ IR Satis 2xis

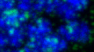
89

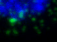


a

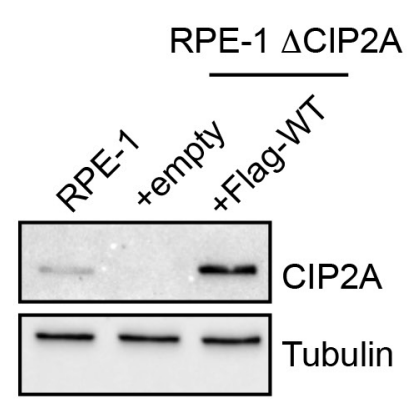

b

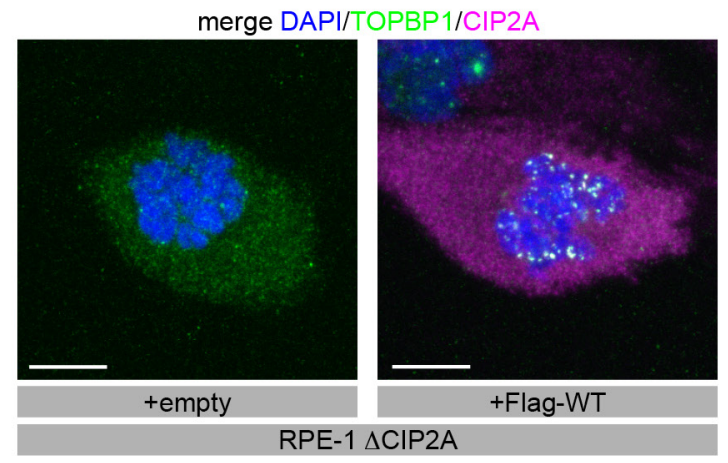

C

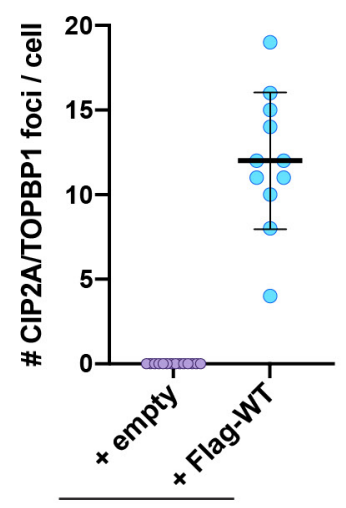

$\overline{\mathrm{RPE}-1 \triangle \mathrm{CIP2A}}$ d

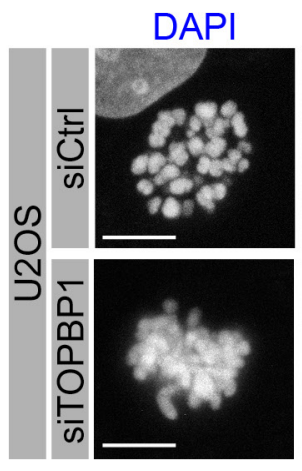

\section{CIP2A}

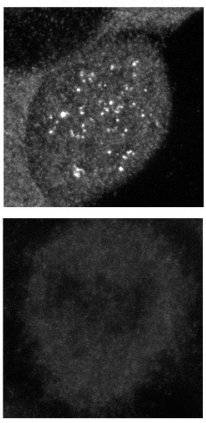

TOPBP1

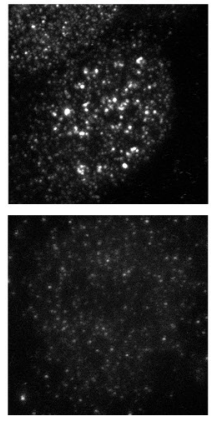

merge

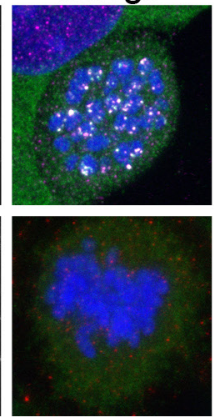

e

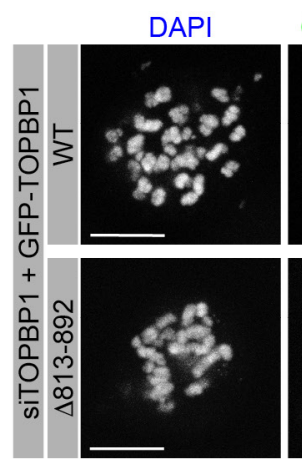

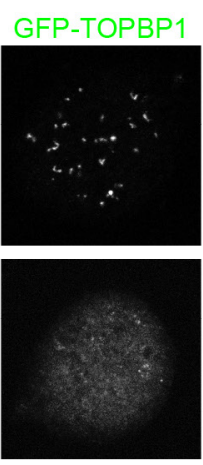
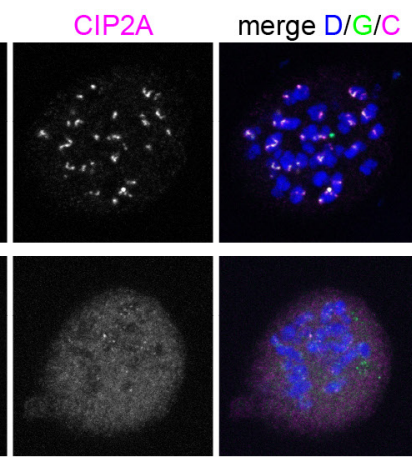

Fig. 3 CIP2A mediates TOPBP1 DSB recruitment in mitosis. a Western blots of total cell extract of parental RPE-1 cells, $\triangle$ CIP2A RPE-1 cells and stably transduced $\triangle$ CIP2A cells (empty vector and vector containing Flag-tagged wild type CIP2A cDNA). b Confocal micrographs (maximum intensity projections) of Nocodazole-arrested empty vector (+Empty) and Flag-tagged CIP2A wild type (+Flag-WT) complemented RPE-1 $\Delta$ CIP2A cells, treated with 1 Gy of IR and stained for TOPBP1 and CIP2A. c Quantification of the experiment in b. Number of CIP2A/TOPBP1 foci per cell was assessed ( $\mathrm{n}=10$ ). d Confocal micrographs (maximum intensity projection) of control siRNA (siCtrl) and TOPBP1 siRNA (siTOPBP1) transfected, Nocodazole-arrested U2OS cells, $1 \mathrm{~h}$ after irradiation with $1 \mathrm{~Gy}$. e Confocal micrographs (maximum intensity projections) of Nocodazole-arrested GFP-TOPBP1 (wild type and $\Delta 813-892$ ) expressing U2OS cells, treated with 1 Gy of IR. Endogenous TOPBP1 was depleted by 5'-UTR targeting TOPBP1 siRNA. All scale bars $=10 \mu \mathrm{m}$. 
bioRxiv preprint doi: https://doi.org/10.1101/2021.02.08.430274; this version posted February 8, 2021. The copyright holder for this preprint (which was not certified by peer review) is the author/funder, who has granted bioRxiv a license to display the preprint in

a
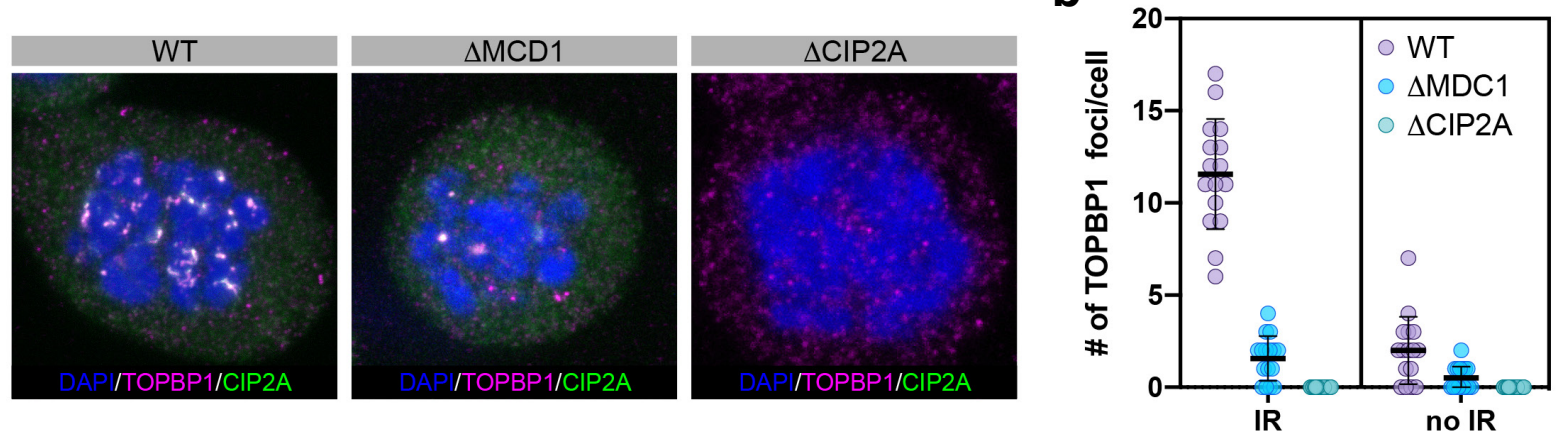

e

c
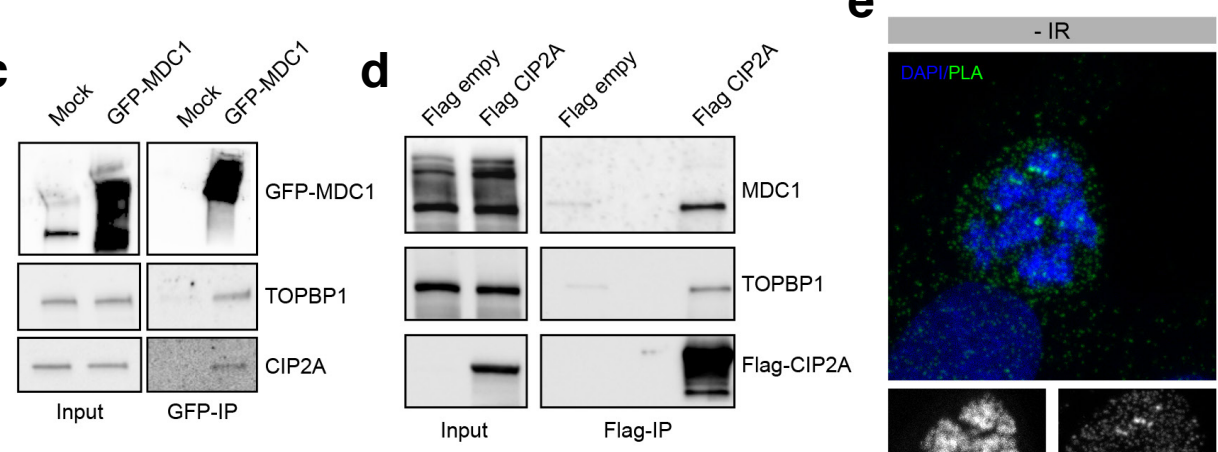

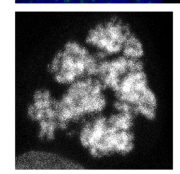

DAPI

f
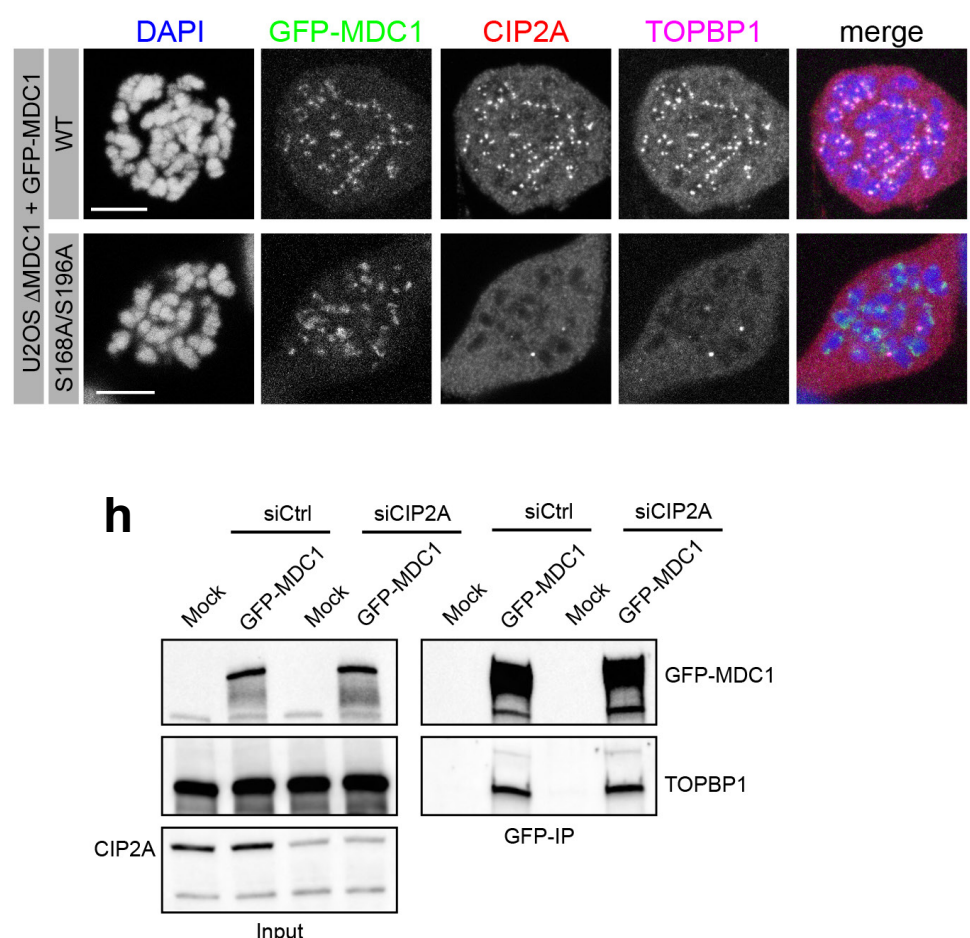

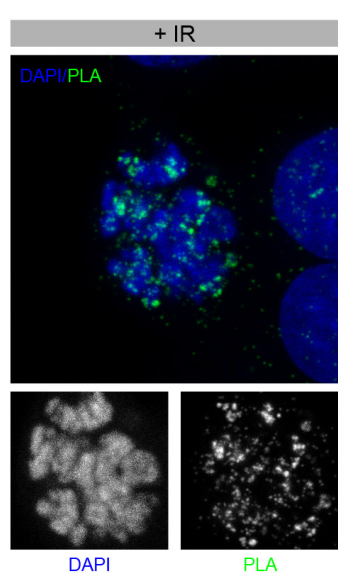

9 Size Number

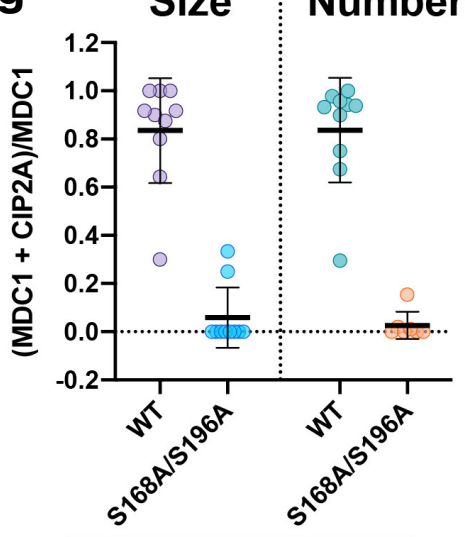

U2OS $\triangle M D C 1+$ GFP-MDC1

Fig. 4 CIP2A-TOPBP recruitment to sites of mitotic DSBs is mediated by MDC1 a Confocal micrographs (maximum intensity projections) of Nocodazole-arrested RPE1 wild type, MDC1 knock-out ( $\triangle \mathrm{MDC} 1)$ and CIP2A knock-out $(\triangle \mathrm{CIP} 2 \mathrm{~A})$ cells, irradiated with $1 \mathrm{~Gy}$ and stained for TOPBP1 and CIP2A. b Quantification of the experiment in a. CIP2A/TOPBP1 foci were manually counted. Each data point represents one mitotic cell $n=16$ per cell line and condition $\mathbf{c}$ GFP pull-downs from 293FT cells transfected with GFP-tagged full-length MDC1. d Flag-immunoprecipitation from 293FT cells transfected with Flag-tagged full-length CIP2A. e Detection of CIP2A-MDC1 co-localization by in situ PLA in U2OS cells arrested in mitosis by Nocodazole and mock treated or treated with $1 \mathrm{~Gy}$ of IR. f Confocal micrographs (maximum intensity projections) of Nocodazole-arrested U2OS $\triangle \mathrm{MDC} 1$ cells stably transfected with GFP-tagged wild type and S168A/S196A mutated MDC1. g Quantitative analysis of GFP-MDC1 and CIP2A co-localization by SQUASSH: Left graph: object size colocalization (area of object overlap divided by total object area). Right graph: object number colocalization (fraction of objects in each channel that overlap $\geq 50 \%$ ). Data points represent individual mitotic cells $(n=9)$. Bars and error bars represent mean and SD. $h$ GFP pull-down from 293FT cells transfected with GFP-tagged full-length MDC1 and either control siRNA (siCtrl) or siRNA against CIP2A (siCIP2A). All scale bars $=10 \mu \mathrm{m}$ 

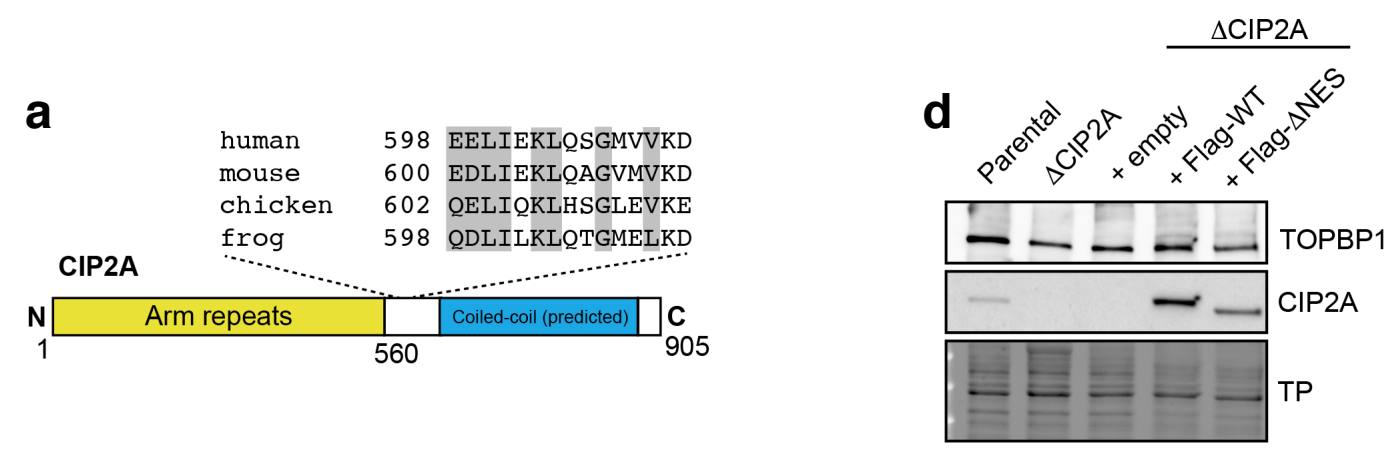

b

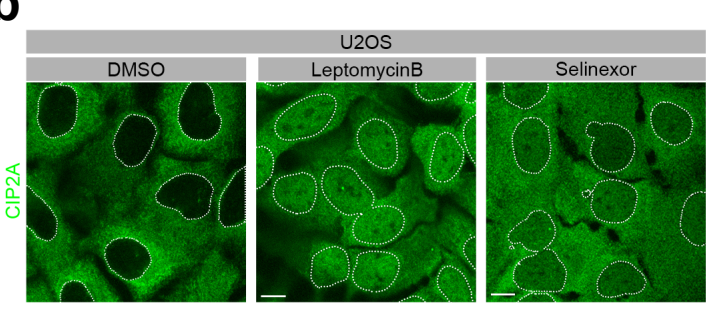

e
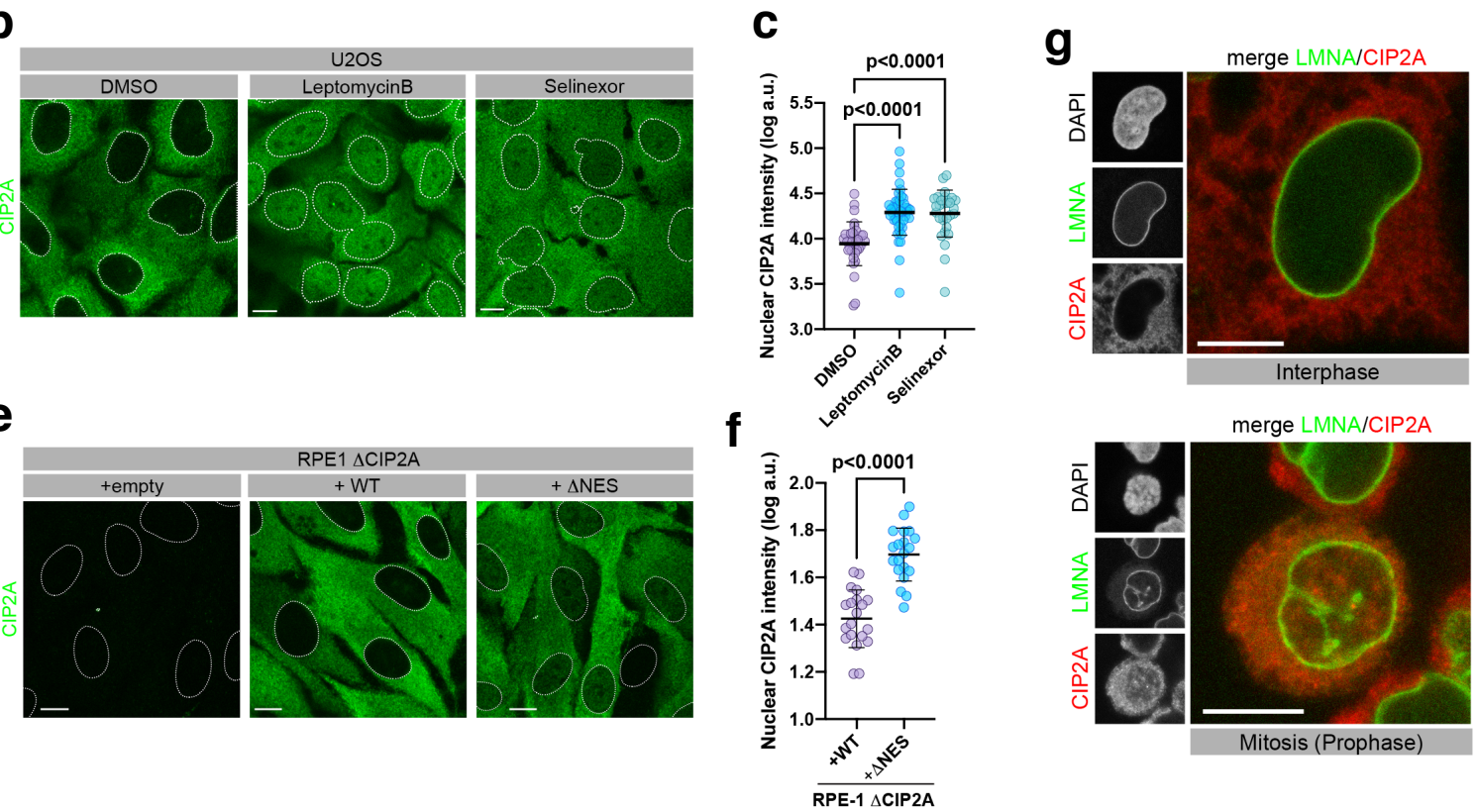

h

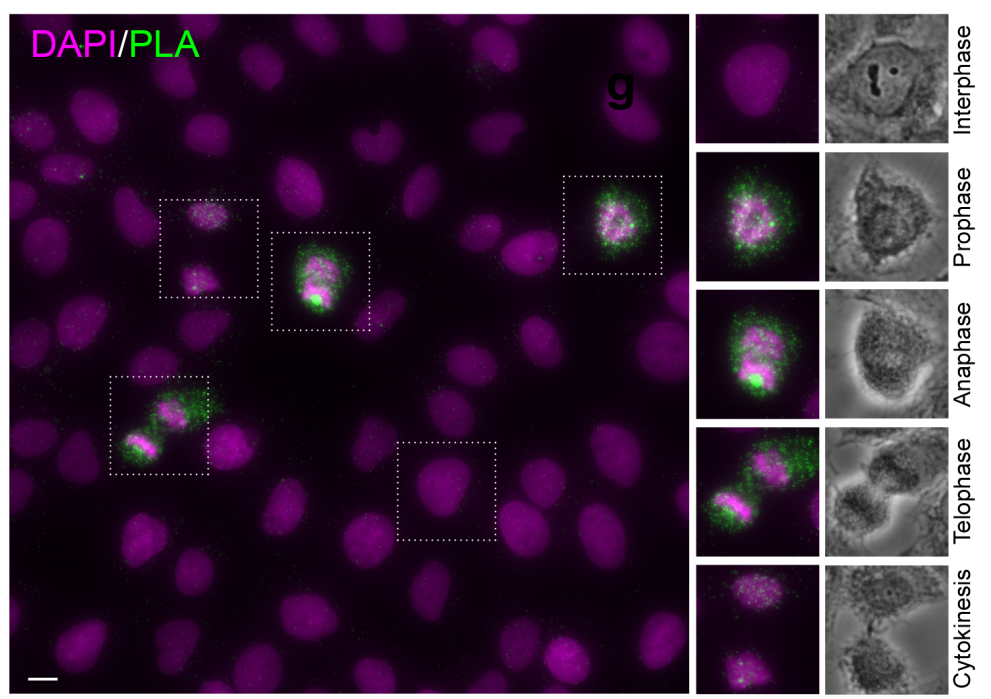

Fig. 5 CRM1-dependent nuclear export sequesters CIP2A from TOPBP1 in interphase cells. a Schematic showing the structural domains of CIP2A and the location of a putative NES. Conserved amino acids matching the NES consensus motif are highlighted. b Confocal micrographs (maximum intensity projections) of Leptomycin B, Selinexor and control DMSO treated U2OS cells, stained for CIP2A. c Quantification of nuclear CIP2A staining of the experiment in a. Statistical significance was calculated using one-way analysis of variance and Sidak's multiple comparison test. Bars and error bars represent mean and SD; DMSO: $n=35$; Leptomycin B: $n=45$; Selinexor: $n=30$. $\mathbf{d}$ Western blots of total cell extract of parental RPE-1 cells, $\triangle$ CIP2A RPE-1 cells and stably transduced $\triangle$ CIP2A cells (empty vector, Flag-tagged wild type CIP2A and Flagtagged CIP2A $\triangle$ NES). TP stands for total protein on blot and serves as loading control. e Confocal micrographs (maximum intensity projections) of RPE1 $\triangle$ CIP2A cells and RPE1 $\triangle$ CIP2A cells stably transduced with Flag-tagged full-length CIP2A and CIP2A deletion mutant (human CIP2A amino acids 561-625; $\triangle N E S$ ), stained for CIP2A. $f$ Quantification of nuclear CIP2A staining of the experiment in e. Statistical significance was calculated using unpaired t-test; bars and error bars represent mean and SD; $n=20$. $\mathbf{g}$ Confocal micrograph (maximum intensity projection) of Hela cells expressing endogenous Clover-tagged LMNA and stained for CIP2A. h Micrograph of in situ PLA, using antibodies against CIP2A and TOPBP1, in unsynchronized and untreated U2OS cells. DAPI staining and phase contrast were used to visualize interphase and four mitotic stages. All scale bars $=10 \mu \mathrm{m}$. 
bioRxiv preprint doi: https://doi org/10.1101/2021.02.08.430274; this version posted February 8, 2021. The copyright holder for this preprint (which was not certified by peer review) is the author/funder, who has granted bioRxiv a license to display the preprint in

a
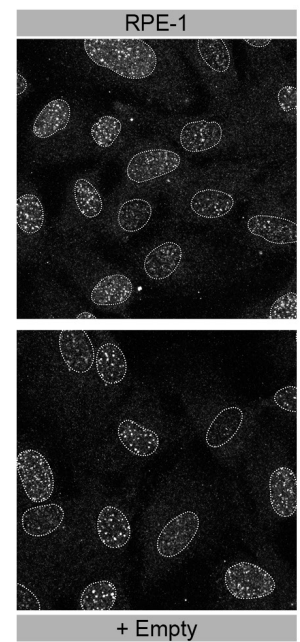

RPE-1 $\triangle$ CIP2A
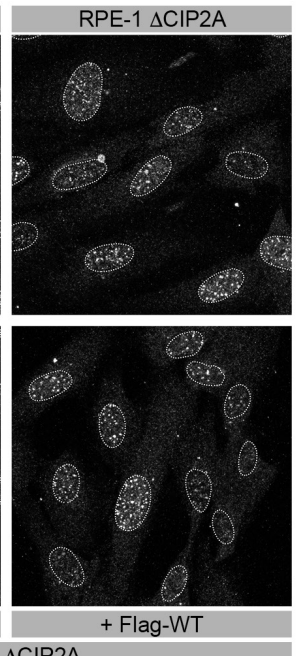

b

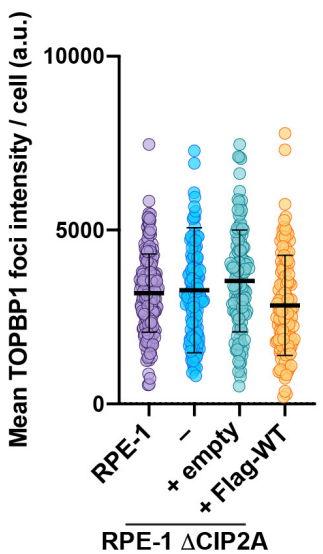

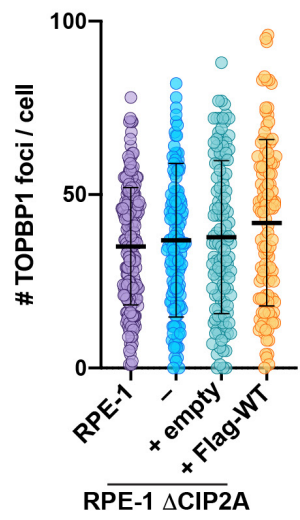

C

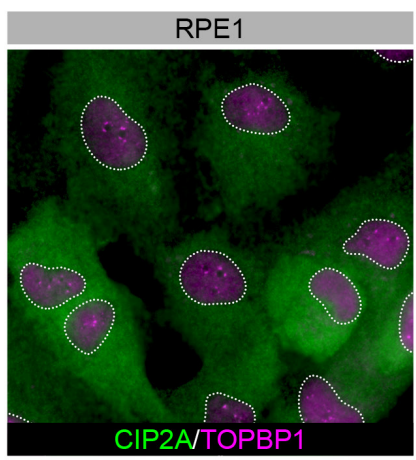

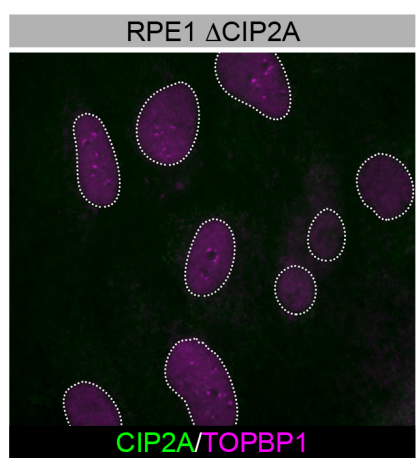

d

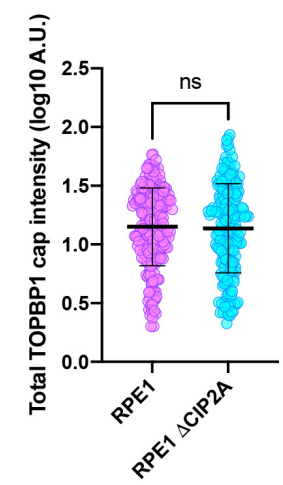

e
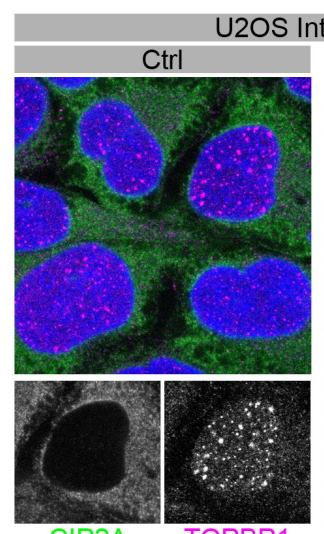

CIP2A

TOPBP1

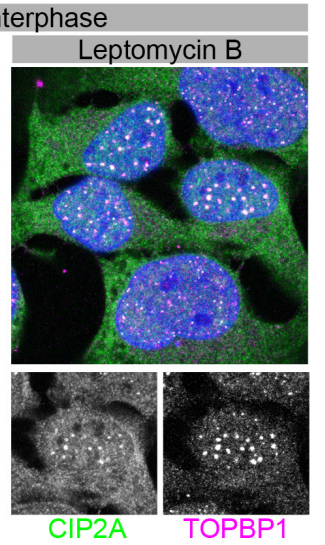

f

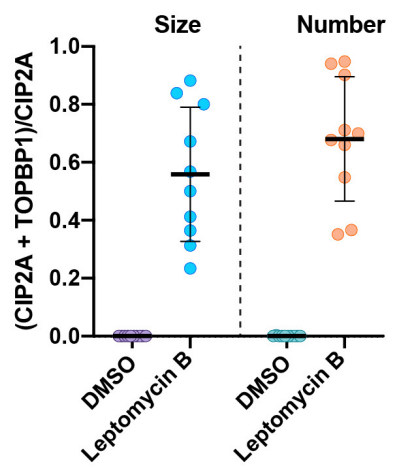

Fig. 6 CIP2A-independent TOPBP1 foci formation in interphase cells. a Confocal micrographs of unsynchronized parental RPE-1 cells, RPE-1 $\triangle$ CIP2A cells and RPE-1 $\Delta$ CIP2A cells stably transduced with empty vector (+Empty) or Flag-tagged full-length CIP2A (+Flag-WT), treated with $3 \mathrm{~Gy}$ of IR and stained for TOPBP1. b Quantification of TOPBP1 foci mean intensity and number of TOPBP1 foci per nucleus in interphase parental RPE1 cells, RPE1 $\triangle$ CIP2A cells and RPE1 $\triangle$ CIP2A cells stably transduced with empty vector (+Empty) or Flag-tagged full-length CIP2A (+Flag-WT). Bars represent mean and SD; RPE-1: $n=200 ; \Delta$ CIP2A: $n=144$; +Empty: $n=128$; +Flag-WT: $n=125$. c Micrographs of RPE1 wild type and $\Delta$ CIP2A cells transfected with I-Ppo1 mRNA to induce rDNA breaks and stained for CIP2A and TOPBP1. d Quantification of the experiment in c. Statistical significance was calculated using unpaired t-test. Bars and error bars represent mean and SD; $n=323$ (RPE1), 329 (RPE1 $\triangle$ CIP2A). ns = not significant. e Confocal micrographs (maximum intensity projections) of U2OS cells pre-treated with DMSO and Leptomycin B, irradiated with $3 \mathrm{~Gy}$ and stained for CIP2A and TOPBP1. $f$ Quantitative analysis of CIP2A and TOPBP1 colocalization of the experiment in e by SQUASSH. Left: object size colocalization (area of object overlap divided by total object area). Right: object number colocalization (fraction of objects in each channel that overlap $\geq$ $50 \%)$. Each data point represents one cell $(n=10)$. Bars and error bars represent mean and SD. All scale bars $=10 \mu \mathrm{m}$. 
bioRxiv preprint doi: https://doi.org/10.1101/2021.02.08.430274; this version posted February 8, 2021. The copyright holder for this preprint (which was not certified by peer review) is the author/funder, who has granted bioRxiv a license to display the preprint in

a perpetuity. It is made available under aCC-BY
no IR $\quad$ IR $6 \mathbf{h}$

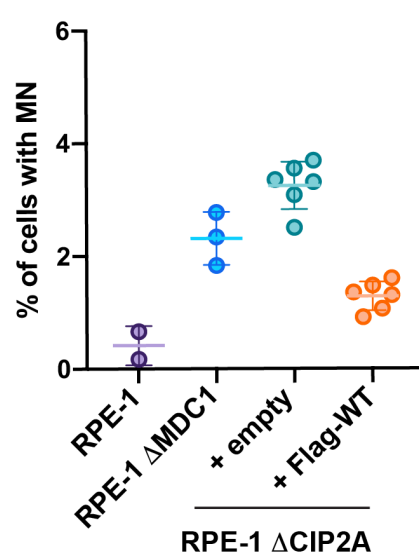

C
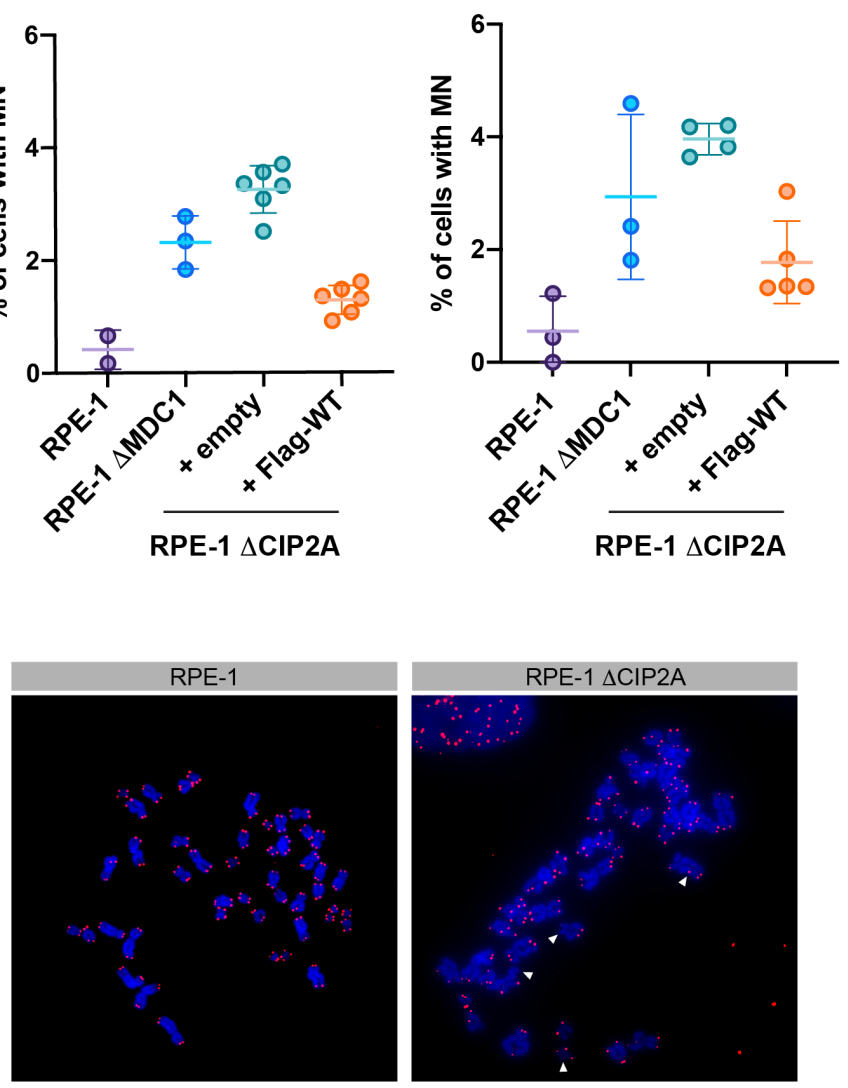

d
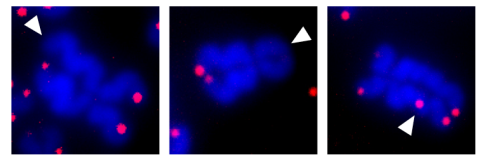

e

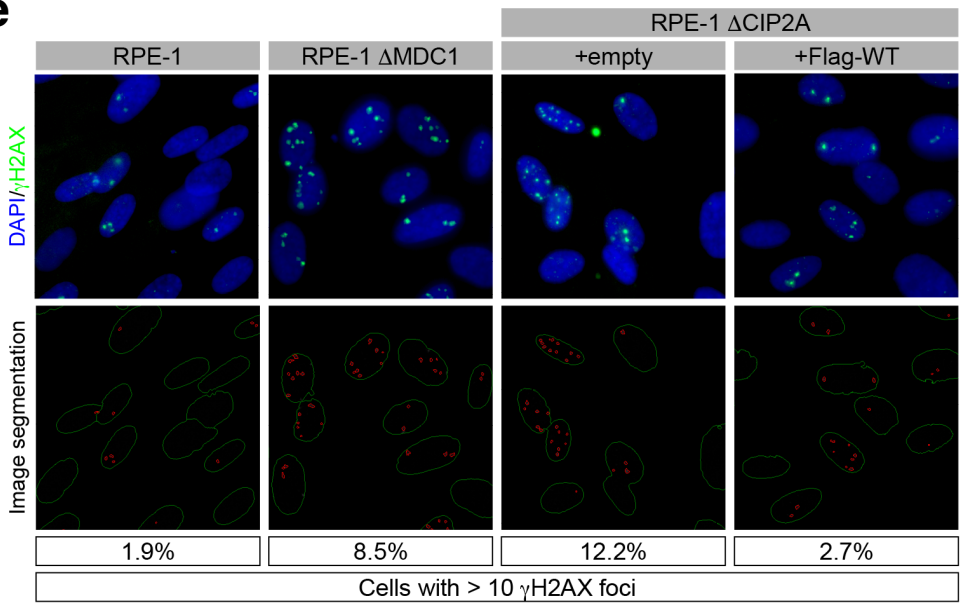

b

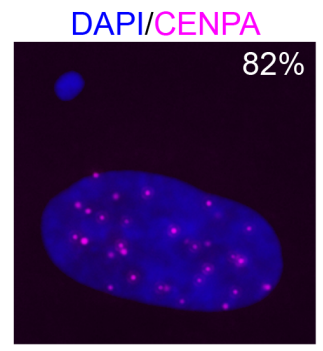

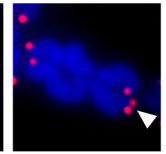
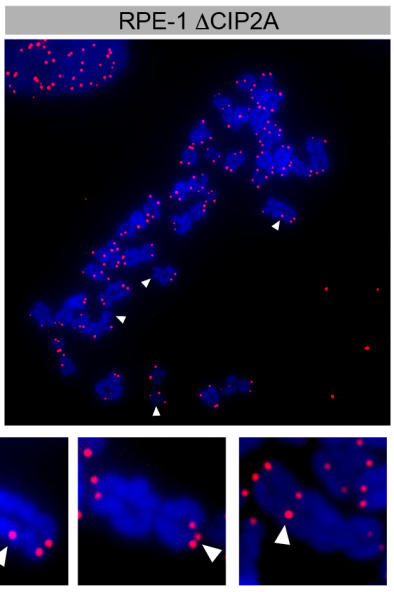

$\mathbf{f}$

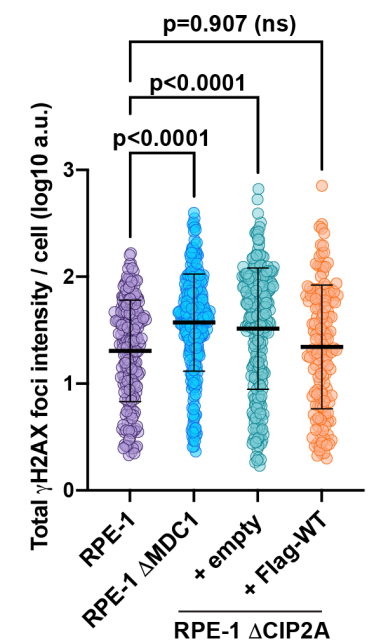

Fig. 7 CIP2A deficient cells display increased micronuclei formation, chromosomal instability and DSB repair defects. a Quantification of micronuclei formation in non-irradiated and irradiated RPE-1 parental cells, RPE-1 $\triangle \mathrm{MDC} 1 \mathrm{cells}$, RPE$1 \triangle$ CIP2A cells stably transduced with empty vector (+ empty) and Flag-tagged wild type CIP2A (+ Flag-WT). b Example of RPE1 $\triangle$ CIP2A cell with micronucleus, stained with CENPA antibody. Percentage of CENPA-negative micronuclei are indicated. $\mathbf{c}$ Examples of chromosomal aberrations in metaphase spreads derived from RPE-1 and RPE-1 $\triangle$ CIP2A cells. Aberrations include single chromatid telomere loss, sister chromatid telomere loss, interstitial telomeres, telomere duplications and dicentric chromosomes. d Quantification of chromosomal aberrations in metaphase spreads derived from untreated RPE-1 parental cells, RPE-1 $\triangle \mathrm{MDC} 1$ cells and RPE-1 $\triangle \mathrm{CIP} 2 \mathrm{~A}$ cells stably transduced with empty vector (+ empty) and Flag-tagged wild type CIP2A (+ Flag-WT). Aberrations were counted manually. Each data point represents one metaphase. $\mathrm{n}=16$ (WT), 12 ( $\triangle \mathrm{MDC} 1$ ), 13 (+Empty), 13 (+Flag-WT) e Residual $\gamma \mathrm{H} 2 \mathrm{AX}$ foci $24 \mathrm{~h}$ after irradiation of RPE-1 parental cells, RPE-1 $\triangle \mathrm{MDC} 1$ cells and RPE-1 $\triangle$ CIP2A cells stably transduced with empty vector (+ empty) and Flag-tagged wild type ClP2A (+ Flag-WT). Cells were arrested in G2 by RO-3306, irradiated with $0.5 \mathrm{~Gy}$ and released from the mitotic arrest. Upper panels: representative micrographs of cells stained for $\gamma \mathrm{H} 2 \mathrm{AX}$. Lower panels: results of image segmentation for quantification. Percentage of cells with $>10 \gamma \mathrm{H} 2 \mathrm{AX}$ foci are indicated. $\mathbf{f}$ Quantification of the experiment in $\mathbf{e}$. Statistical significance was calculated using one-way analysis of variance and Sidak's multiple comparison test. Bars and error bars represent mean and SD; RPE1: $n=368 ; \Delta M D C 1$ : $n=330 ;+$ Empty: $n=219$; +Flag-WT: $n=224$. All scale bars=10 $\mu$ m. 